\title{
Servo-Stabilization of Low-Frequency Oscillations in a Liquid Bipropellant Rocket Motor
}

\author{
FRANK E. MARBLE ${ }^{1}$ and DALE W. COX, JR. ${ }^{2}$ \\ Daniel and Florence Guggenheim Jet Propulsion Center, California Institute of Technology, Pasadena, Calif.
}

\begin{abstract}
The recent work of $H$. S. Tsien concerning the servostabilization of rocket motors is extended to the liquid bipropellant rocket motor. It is shown that by use of a feedback system containing a device to sense the combustion chamber pressure, a suitably designed amplifier, and a servomechanism which governs the propellant flow, the low-frequency oscillations which occur in the rocket configuration may be stabilized for any value of combustion time lag. A method is given for determining a transfer function of the feedback loop which will assure stable operation. The technique of the Satche diagram is employed in stability analysis.
\end{abstract}

\section{Introduction}

T HE low-frequency instability of liquid propellant rocket motors has been attributed by Gunder and Friant (1), ${ }^{3}$ Yachter (2), Summerfield (3), and Crocco (4) to the coupled oscillations of rocket chamber pressure and of flow rates of propellant in the feed lines. The instability arises from the fact that propellant combustion lags injection, due to the heat transfer required for propellant evaporation, time required for chemical reaction, etc., thereby introducing a phase difference between the oscillations of the two systems. The most general of these analyses is that of Crocco which treats both monopropellant and bipropellant systems and gives some physical basis for the introduction of a time lag. This latter aspect has since been generalized by Tsien (5).

Various possibilities have been discussed for removing the low-frequency instability through suitable choice of rocket configuration parameters, such as the propellant pressure drop across the injector nozzle. In some cases these changes may be undesirable or impossible; for example, in a rocket required to have a variable thrust, it may prove difficult to stabilize for all conditions of operation. Tsien (5) has investigated the more general concept of removing combustion chamber instability by use of a feedback servo control which senses the oscillating pressure in the combustion chamber and changes the propellant flow by the proper amount and in the proper phase to damp the oscillation. In the same paper the feasibility of such a system is demonstrated for the monopropellant rocket. For the purpose of this demonstration, Tsien

Received October 1, 1952.

1 Assistant Professor.

${ }^{2}$ Graduate Student; .LCDR, U. S. Navy.

${ }^{3}$ Numbers in parentheses refer to the References on page 81 . introduced a graphical method of stability analysis, based on a suggestion by Satche (6), which is the appropriate technique for analyzing systems with time lag in the same sense that the familiar Nyquist diagram is appropriate for systems without time lag. Using the Satche diagram, Tsien was able to place quite general restrictions on the feedback loop which would assure stable rocket operation.

The present paper aims to extend these considerations to the bipropellant rocket and to find criteria on the feedback loop to provide stable operation. The authors are deeply grateful to Professor H. S. Tsien for initiating this problem and for his active interest during its progress.

\section{Dynamies of the Bipropellant Rocket}

The dynamics of the bipropellant rocket chamber, Fig. 1, are more complicated than those of the monopropellant rocket, due to the additional degree of freedom associated with the second propellant line and to the periodic changes of combustion gas temperature caused by variations in mixture ratio. Because only low-frequency oscillations are under consideration, the pressure at any time will be assumed uniform over the combustion chamber.

If the dependent variables of the system are taken to be the chamber pressure, the oxidizer flow rate, and the fuel flow rate, three equations may be written relating these quantities to each other and to the physical configuration. They are the continuity relation for the combustion chamber, and two conditions of dynamic equilibrium for the fluid in the oxidizer and fuel lines.

Following Crocco's notation (4), let $M_{g}$ be the mass of burned gas in the combustion chamber, $\dot{m}_{b}$ the mass rate of burning, and $\dot{m}_{e}$ the mass rate of ejection from the discharge nozzle. Then the relation $d / d t\left(M_{g}\right)=\dot{m}_{b}-\dot{m}_{c}$ expresses

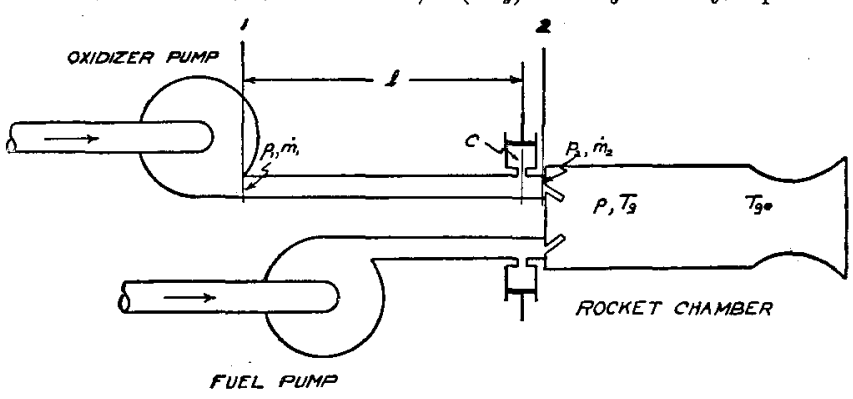

FIG. 1 SCHEMATIC DIAGRAM OF BIPROPELLANT ROCKET AND PROPELLANT FEED SYSTEM 
the conservation of mass. Suppose the state of the dynamic system is always close to an equilibrium value defined by $\bar{M}_{g}, \overline{\dot{m}}_{b}=\overline{\dot{m}}_{e}=\overline{\dot{m}}$, then the residence time which, on the average, the burned gas spends in the chamber, is $\vartheta_{g}=$ $\bar{M}_{g} / \dot{m}$. Anticipating the use of perturbation analysis, the fractional variation of burning rate and discharge rate may be introduced, $\mu_{b}=\frac{\dot{m}_{b}-\overline{\dot{m}}}{\overline{\dot{m}}}$ and $\mu_{e}=\frac{\dot{m}_{e}-\overline{\dot{m}}}{\overline{\dot{m}}}$ together with a dimensionless time $z=t / \vartheta_{o}$ to write the mass conservation as

$$
\frac{d}{d z}\left(\frac{M_{g}}{\bar{M}_{o}}\right)=\mu_{b}
$$

The propellant burning rate $\dot{m}_{b}$ differs from the total propellant injection rate $\dot{m}_{i}$ because of the combustion time lag. The time $\tau$ required for the transformation of propellant into products of combustion may be represented by means of the integral

$$
\int_{i-\tau}^{t} f\left(p, T_{o}\right) d t^{\prime}=C=\text { const } \ldots \ldots \ldots \ldots
$$

where $f\left(p, T_{g}\right)$ is a function depending upon the mechanics of the process. The chief quantities upon which this function depends are $p$, the uniform chamber pressure, and $T_{g}$, the ambient gas temperature in the neighborhood of the injector. The meaning of this integral may be clarified somewhat by thinking of $f$ to be, for example, the rate of heat transfer to the liquid propellant, a certain total quantity $C$ of heat being required before the transformation is complete. The principal quantities associated with the heat transfer process are the ambient temperature and pressure so that the time $\tau$ required for transformation will be large or small depending upon whether the heat transfer rate is small or large.

Now if the time lag were independent of time, the fractional variation of burning rate $\mu_{b}$ would be identical with the fractional variation of injection rate measured at the time $\tau$ earlier. Denoting the dimensionless time lag $\tau / \vartheta_{g}=\delta$, constant time lag would imply $\mu_{b}(z)=\mu_{i}(z-\delta)$. However, if the time lag is increasing during the process, the burning rate is depressed below the injection rate in proportion to the product of the propellant flow rate and the rate of change of time lag. Therefore, using dimensionless quantities and neglecting a term of second order in the small variations

$$
\mu_{b}(z) \approx \mu_{i}(z-\delta)-\frac{d \delta}{d z}=0
$$

The dependence of the time lag upon time is, in reality, governed by the integral, Equation [2], and differentiating this with respect to time gives

$$
f\left[p(t), T_{\theta}(t)\right]-f\left[p(t-\tau), T_{\vartheta}(t-\tau)\right]\left(1-\frac{d \tau}{d t}\right) \ldots
$$

Since it is assumed that the chamber pressure and ambient temperature vary only slightly from their steady-state values $\bar{p}$ and $\bar{T}_{q}$, the values of $f$ may be approximated, following Tsien (5), through a Taylor expansion about $p=\bar{p}, T_{g}=\bar{T}_{g}$

$$
\begin{aligned}
& f\left[p(t), T_{g}(t)\right] \approx f\left(\bar{p}, \bar{T}_{g}\right)+\frac{\partial f\left(\bar{p}, \bar{T}_{g}\right)}{\partial \bar{p}}[p(t)-\bar{p}]+ \\
& \frac{\partial f\left(\bar{p}, \bar{T}_{g}\right)}{\partial \bar{T}_{g}}\left[T_{o}(t)-\bar{T}_{g}\right] \\
& f\left[p(t-\tau), T_{g}(t-\tau)\right] \approx f\left(\bar{p}, \bar{T}_{g}\right)+\quad \\
& \quad \frac{\partial f\left(\bar{p}, \bar{T}_{g}\right)}{\partial \bar{p}}[p(t-\tau)-\bar{p}]+\frac{\partial f\left(\bar{p}, \bar{T}_{g}\right)}{\partial \bar{T}_{g}}\left[T_{g}(t-\tau)-\bar{T}_{g}\right]
\end{aligned}
$$

Upon substituting these relations into Equation [4] and deleting second-order differences, the variation of time lag may be expressed
For a monopropellant rocket the combustion gas temperature is nearly constant so that the second term on the right side of Equation [5] vanishes. Calling $\partial \log f\left(\bar{p}, \bar{T}_{a}\right) / \partial \log \bar{p} \equiv n$, Tsien's generalization of Crocco's treatment is obtained. For the bipropellant rocket the combustion gas temperature may experience considerable variation, resulting from the variation of propellant mixture ratio, and consequently another parameter $\partial \log f\left(\bar{p}, \bar{T}_{\vartheta}\right) / \partial \log \bar{T}_{\sigma} \equiv m$ enters the problem. Knowledge of combustion lag mechanics is so meager, however, that rational estimate of its importance is difficult. Consequently, as in previous investigations, the dependence of combustion time lag upon gas temperature will be neglected. Then, denoting the fractional variation in chamber pressure as $\varphi \equiv(p-\bar{p}) / \bar{p}$, the expression for dimensionless time lag variation is

$$
\frac{d \delta}{d z} \approx n[\varphi(z-\delta)-\varphi(z)] \ldots \ldots \ldots \ldots \ldots
$$

The fractional variation in total propellant injection rate $\mu_{i}$, which occurs in Equation [3], is most conveniently expressed in terms of the individual fractional variations of oxidizer flow, $\mu_{0} \equiv\left(\dot{m}_{0}-\overline{\dot{m}}_{0}\right) / \dot{\dot{m}}_{0}$, and of fuel flow, $\mu_{f} \equiv\left(\dot{m}_{f}-\overline{\dot{m}}_{f}\right) / \dot{m}_{f}$, and the steady-state mixture ratio $\bar{\gamma} \equiv \overline{\dot{m}}_{0} / \overline{\dot{m}}_{f}$. Again following Crocco in defining $H \equiv \bar{r}-1 / 2(\vec{R}+1)$, depending on the steady-state mixture ratio, it is an elementary calculation to show that, to the first order,

$$
\mu_{i}=(1 / 2+H) \mu_{0}+(1 / 2-H) \mu_{f}
$$

Consequently, the propellant burning rate is just

$$
\begin{aligned}
\mu_{b}(z)=\left(\frac{1}{2}+H\right) \mu_{0}(z-\delta)+\left(\frac{1}{2}-H\right) \mu_{j}(z-\delta)- \\
n[\varphi(z-\delta)-\varphi(z)] \ldots
\end{aligned}
$$

In calculating the variation of exit nozzle flow, $\dot{m}_{e}$, it is convenient to consider the nozzle flow as quasi-steady, that is, the flow corresponds to a time sequence of steady equilibrium flow patterns. Then the ratio of instantaneous to steady mass discharge rate is

$$
\frac{\dot{m}_{c}}{\overline{\dot{m}}}=\frac{p}{\bar{p}}\left(\frac{\bar{T}_{g}}{T_{\mathrm{ge}}}\right)^{3 / 2}
$$

where $T_{g e}$ is the gas temperature at the exhaust end of the combustion chamber. Tsien has shown (7) that the quasisteady approximation is adequate for low-frequency instability under discussion here.

For the monopropellant rocket, where $T_{g e}$ is invariable, the mass discharge rate depends directly upon the combustion chamber pressure $p$, and the fractional variation of mass discharge rate is simply $\mu_{e}=\varphi$. Where the variation of mixture in a bipropellant rocket causes appreciable variation of temperature, this too must, according to Equation [8], be accounted for in computing the rate of gas discharge. Suppose the gas temperature at the exhaust end is $T_{o e}$ at the time $t$. Recalling that the gas temperature depends upon only the propellant mixture ratio, the temperature $T_{g e}$ can be related to the injection rates of propellant which formed the gas volume. The generation of gas took place at a time $\vartheta_{g}$ earlier than the gas appeared at the nozzle end of the chamber, that is, at a time $t-\vartheta_{g}$. Furthermore, the time required between injection of the propellants and generation of the gas is the time lag $\tau$. Therefore the gas temperature at the exhaust end of the chamber is that corresponding to the mixture ratio at the time $t-\vartheta_{\theta}-\tau$, or at the dimensionless time $z-1-\delta$.

Now if $r$ is the instantaneous mixture ratio, the change of gas temperature from its equilibrium value is, approximately, $\left(d T_{o} / d r\right)_{r}^{-} \cdot(r-\bar{r})$. Furthermore, the variation of mixture ratio from its equilibrium value follows directly from the definition $r=\dot{m}_{0} / \dot{m}_{f} \approx \tilde{r}\left(1+\mu_{0}-\mu_{f}\right)$. Denoting the di-

$$
\frac{d \tau}{d t}=\frac{\partial \log f\left(\bar{p}, \bar{T}_{g}\right)}{\partial \log \bar{p}}\left[\frac{p(t-\tau)-p(t)}{\bar{p}}\right]+\frac{\partial \log f\left(\bar{p}, \breve{T}_{\theta}\right)}{\partial \log \bar{T}_{o}}\left[\frac{T_{g}(t-\tau)-T_{o}(t)}{\bar{T}_{\theta}}\right]
$$


mensionless slope of the gas temperature curve, $\left(\bar{r} / \bar{T}_{g}\right)\left(d T_{g} /\right.$ $d r)_{r}^{-}$, by $2 K$, the effect of combustion gas temperature upon fractional variation of mass discharge is $K\left[\mu_{0}(z-\delta-1)-\mu_{f}(z-\right.$ $\delta-1)]$. Thus the complete expression is

$$
\mu_{e}(z)=\varphi(z)-K\left[\mu_{0}(z-\delta-1)-\mu_{f}(z-\delta-1)\right] \ldots \text { [9] }
$$

Finally, the time variation of gas storage within the combustion chamber is shown by Crocco to be

$$
\begin{aligned}
& \frac{d}{d z}\left(\frac{M_{g}}{\bar{M}_{a}}\right) \approx \frac{d \varphi}{d z}+ \\
& 2 K\left\{\left[\mu_{0}(z-\delta-1)-\mu_{f}(z-\delta-1)\right]-\left\{\mu_{0}(z-\delta)-\mu_{f}(z-\delta)\right]\right\}
\end{aligned}
$$

It is easy to trace the origin of the terms entering the right side of this expression. When the temperature is constant, the variation of mass in the chamber is directly proportional to the fractional variation of the uniform pressure, that is, to $d \varphi / d z$. When the temperature is not constant, a term must appear to account for the fact that the gas leaving the discharge, generated at $z-\delta-1$, is of different temperature and density from that generated at the injector, at $z-\delta$. Conversion of these temperature differences to the corresponding differences in oxidizer and fuel rates leads to the second term on the right side of Equation [10].

Introducing Equations [7], [9], and [10] in the original expression for the mass conservation in the combustion chamber, Equation [1], gives this relation in terms of the desired dependent variables

$$
\begin{aligned}
& \frac{d \varphi}{d z}+(1-n) \varphi+n_{\varphi}(z-\delta)+ \\
& {\left[K_{\mu_{0}}(z-\delta-1)-2 K_{\mu_{0}}(z-\delta)-\left(\frac{1}{2}+H\right) \mu_{0}(z-\delta)\right]-} \\
& {\left[K_{\mu_{f}}(z-\delta-1)-2 K_{\mu_{f}}(z-\delta)+\left(\frac{1}{2}-H\right) \mu_{f}(z-\delta)\right]=0}
\end{aligned}
$$

Description of the system may be completed by relating the individual propellant flow rates to the mechanical properties of the feed system. Consider each propellant feed system to consist, Fig. 1, of a feeding mechanism (e.g., turbopump), a propellant supply line, an injector nozzle, and a variable capacity situated in the line so close to the injector nozzle that the inertia of fluid between the capacity and the injector nozzle may be neglected. Since the process is the same in oxidizer and fuel lines, notation differentiating the two lines will be postponed.

Denote by subscript 1 the conditions in the propellant line just downstream of the pump, and by 2 the conditions just ahead of the injector. Following Tsien (5), the variation of $\dot{m}_{1}$, the pump discharge rate, from its equilibrium value may be written

$$
\frac{\dot{m}_{1}-\overline{\dot{m}}_{1}}{\overline{\dot{m}}_{1}}=-\alpha \frac{p_{1}-\bar{p}_{1}}{\bar{p}_{1}} \ldots \ldots \ldots \ldots
$$

When $\alpha=0$, this relation indicates no response of mass flow to a change of line pressure, that is, a positive displacement system. Conversely, when $\alpha=\infty$, no change of line pressure may be effected by changing the flow rate, which is characteristic of the constant pressure feed or pressurized tank. Intermediate values of $\alpha$ correspond to particular types of pump, and if the response time of the turbopump system is very long with respect to the period of chamber pressure oscillation, the pump will operate at constant speed and $-\alpha$ corresponds to the slope of the pump characteristic (Fig. 2).

The pressure difference $p_{1}-p$ along the propellant lines is due primarily to the liquid acceleration through the injector nozzle and the inertial reaction of the fluid column in the propellant line. The frictional loss will be neglected. If $S$ is the area of the propellant jet issuing from the injector, and $\rho$ the propellant density, the pressure difference $p_{2}-p$ across the injector is $\dot{m}^{2} / 2 \rho S^{2}$. Furthermore, if $l$ and $A$ are the length and cross-sectional area of the connecting line, the inertial pressure difference follows from the momentum equation $\left(p_{1}-p\right) A=d / d t\left(l \dot{m}_{1}\right)$. The mass flow $\dot{m}$ through the injector may differ from the mass flow $\dot{m}_{1}$ in the connecting line because of the variable capacitance situated between the two. Since the propellant is considered incompressible, the difference of the two values of mass flow may be expressed $\dot{m}-\dot{m}_{1}=d C / d t$, where $C$ is proportional to the instantaneous volume of the eapacitance. This capacitance has the dimension of mass per unit time $X$ time and is conveniently represented in the dimensionless form $k \equiv C / \bar{m} \vartheta_{g}$. Then if the pressure drop across the propellant injector under steady operation, $\bar{m}^{2} / 2 \rho S^{2}$, is denoted $\Delta \bar{p}$, two dimensionless parameters may be introduced: the inertia of the connecting line $J \equiv l_{\dot{m}} / 2 \Delta \bar{p} A \vartheta_{a}$, and the injector pressure ratio $P \equiv \bar{p} / 2 \Delta \bar{p}$ Finally eliminating variable conditions at the pump and at the capacitance, the dynamic equilibrium of a propellant line may be written

$J \frac{d \mu}{d z}+\left[\frac{1}{\alpha}\left(P+\frac{1}{2}\right)+1\right] \mu+P_{\varphi}+J \frac{d^{2} k}{d z^{2}}+\frac{1}{\alpha} \frac{d k}{d z}=0$

An equation of this type holds independently for the oxidizer and fuel lines.

\section{Stability of the Bipropellant Rocket}

When the propellant lines are of fixed capacity, the system is described by three independent variables, $\varphi(z), \mu_{0}(z)$, and $\mu_{f}(z)$ representing the fractional variations in chamber pressure, oxidizer, and fuel flow. The three differential equations are, for the combustion chamber

$$
\begin{aligned}
& \frac{d \varphi}{d^{2} z}+(1-n)_{\varphi}+n \varphi(z-\delta)+ \\
& {\left[K_{\mu_{0}}(z-\delta-1)-2 K_{\mu_{0}}(z-\delta)-\left(\frac{1}{2}+H\right) \mu_{0}(z-\delta)\right]-} \\
& {\left[K_{\mu_{f}}(z-\delta-1)-2 K_{\mu_{f}}(z-\delta)+\left(\frac{1}{2}-H\right) \mu_{f}(z-\delta)\right]=0}
\end{aligned}
$$

and for the oxidizer and fuel lines

$$
\begin{aligned}
& J_{0} \frac{d \mu_{0}}{d z}+\left[\frac{1}{\alpha_{0}}\left(P_{0}+\frac{1}{2}\right)+1\right] \mu_{0}+P_{0 \varphi}=0 \\
& J_{f} \frac{d \mu_{f}}{d z}+\left[\frac{1}{\alpha_{f}}\left(P_{f}+\frac{1}{2}\right)+1\right] \mu_{f}+P_{f \varphi}=0 \ldots
\end{aligned}
$$

The stability or instability of the system, that is, whether the amplitude of particular oscillatory solutions will diminish or

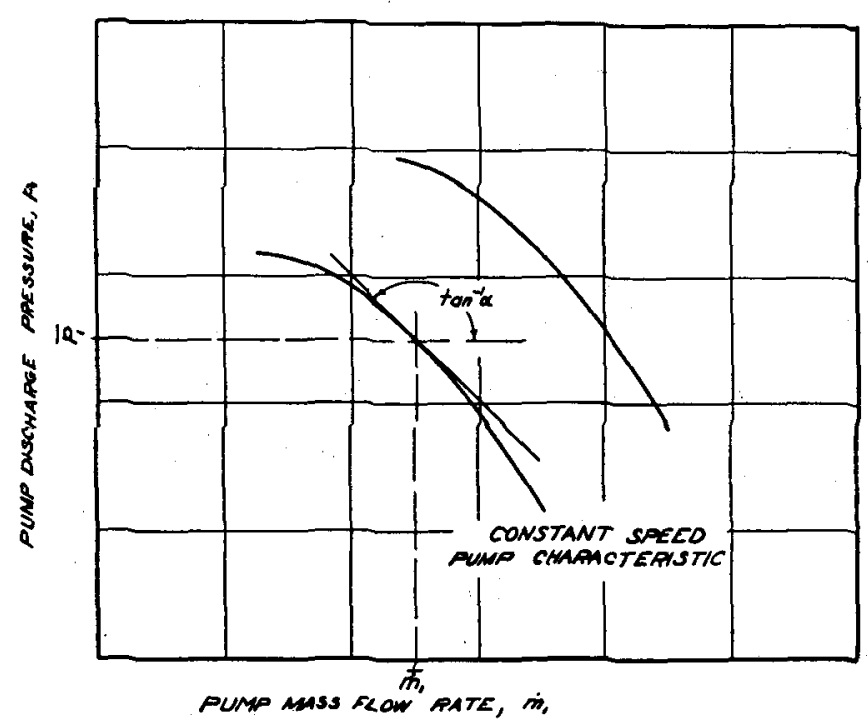

Fig. 2 aPPROXIMATION TO CONSTANT SPEED PUMP CHARACTERISTIC 
grow, is most easily discussed through introducing the Laplace transform, defined as

$$
\Phi(s)=\int_{0}^{\infty} \varphi(z) e^{-s z} d z \ldots \ldots \ldots \ldots \ldots
$$

for the variable $\varphi(z)$. Similarly defining $M_{0}(s)$ and $M_{f}(s)$ as the Laplace transforms of $\mu_{0}(z)$ and $\mu_{f}(z)$, each of the differential equations may be transformed, as shown in Appendix 1, into inhomogeneous algebraic equations in the complex variable $s$. The inhomogeneous portion depends upon the initial conditions of the problem and hence is associated with the solution of transient problems. The corresponding homogeneous equations

$$
\begin{gathered}
{\left[s+1-n+n e^{-s \delta}\right] \Phi(s)+} \\
e^{-s \delta}\left[K e^{-s}-\left(2 K+H+\frac{1}{2}\right)\right] M_{0}(s)- \\
e^{-s \delta}\left[K e^{-s}-\left(2 K+H-\frac{1}{2}\right)\right] M_{f}(s)=0 \\
P_{0} \Phi(s)+\left[J_{0} s+\frac{1}{\alpha_{0}}\left(P_{0}+\frac{1}{2}\right)+1\right] M_{0}(s)=0 \\
P_{f} \Phi(s)+\left[J_{f} s+\frac{1}{\alpha_{f}}\left(P_{f}+\frac{1}{2}\right)+1\right] M_{f}(s)=0 \ldots[17]
\end{gathered}
$$

describe the free oscillations which are of interest in the stability problem. For nontrivial solutions the determinant of the coefficients of Equations [17] must vanish, that is

$$
\begin{array}{cc}
s+(1-n)+n e^{-s \delta} & {\left[K e^{-s}-\left(2 K+H+\frac{1}{2}\right)\right] e^{-s \delta}} \\
P_{0} & J_{0} s+1+\frac{1}{\alpha_{0}}\left(P_{0}+\frac{1}{2}\right) \\
P_{f} & 0
\end{array}
$$

Equation [18] is a transcendental equation in the complex variable $s$, the roots of which determine the stability of rocket chamber pressure oscillations: If any root of $G^{\prime}(s)=0$ posseses a positive real part, the system is unstable. To check on the existence of such roots, it is possible to apply the familiar Nyquist criterion which traces the course of the complex function $G^{\prime}(s)$ as $s$ describes a counterclockwise contour in the right half of the s-plane consisting of the imaginary axis and a large semicircle in the right half plane. Then the negative change in argument of $G^{\prime}(s)$ is the difference in number of zeros and poles of $G^{\prime}(s)$ in the right half s-plane. Graphically this number appears as the number of complete revolutions made about the origin by the trace of $G^{\prime}(s)$. If the number of poles is known independently, the number of zeros in the right half $s$-plane is known and the stability of the system is determined.

The present problem is complicated by the time lag $\delta$, which not only makes calculation of $G^{\prime}(s)$ laborious but accentuates the fact that the value of $\delta$ is usually not known accurately. To improve this situation, Tsien (5) has introduced another technique based upon a suggestion of Satche (6), which separates the function $G^{\prime}(s)$ into two parts, the first containing $e^{-s \delta}$ as a factor, the second independent of the time lag. In the present example the separation may be affected by noting that the Equation [18] may be expressed as the combination of two determinants

$$
D_{1}(s)=\left|\begin{array}{ccc}
s+1-n & 0 & 0 \\
P_{0} & \frac{1}{\alpha_{0}}\left(P_{0}+\frac{1}{2}\right) & 0 \\
& 0 & \frac{1}{\alpha_{f}}\left(P_{f}+\frac{1}{2}\right)
\end{array}\right| \ldots[19]
$$

$$
\left.D_{2}(s)=\mid \begin{array}{ccc}
n & \begin{array}{c}
K e^{-s}- \\
\left(2 K+H+\frac{1}{2}\right)
\end{array} & -\left[K e^{-s}-\right. \\
& \left(2 K+H-\frac{1}{2}\right)
\end{array}\right]
$$

such that Equation [18] may be written

$$
\mathfrak{D}_{1}(s)+e-s \delta D_{2}(s)=0 .
$$

Following Tsien in calling $g_{1}(s) \equiv e^{-s \delta}$ and defining $g_{2}(s) \equiv$ $-\left(D_{1}(s) / D_{2}(s)\right)$, the characteristic equation becomes

$$
g_{1}(s)-g_{2}(s) \equiv G(s)=0
$$

where the complex function $G(s)$ is represented as the difference of the two vectors $g_{1}(s)$ and $g_{2}(s)$.

Assume for the moment that $G(s)$ has no poles in the right half plane. Then if $s$ describes its previous contour, the number of zeros which $G(s)$ has in the right half plane is equal to the number of complete clockwise rotations made by a vector having its head on the trace of $g_{1}(s)$ and its tail on the trace of $g_{2}(s)$. Since all values of $g_{1}(s)$ lie on or within the unit circle, the vector $G(s)$ can make a complete rotation if the

$$
\begin{array}{c|c}
-\left[K e^{-s}-\left(2 K+H-\frac{1}{2}\right)\right] e^{-s \delta} & \\
0 & \equiv G^{\prime}(s)=0 \\
J_{f} s+1+\frac{1}{\alpha_{f}}\left(P_{f}+\frac{1}{2}\right) & \ldots[18]
\end{array}
$$

trace of $g_{2}(s)$ either passes through the unit circle or encircles the origin. Conversely, if the trace of $g_{2}(s)$ lies outside the unit circle and does not encircle the origin, the system is definitely stable.

Actually, the function $G(s)$ may possess poles in the right half plane, due to zeros of the determinant $D_{2}(s)$ which constitutes the denominator of $g_{2}(s)$. Thus the number of clockwise turns which $g_{2}(s)$ makes about the origin must be modified by adding the number of poles induced through the roots of $\mathfrak{D}_{2}(s)$. This number of poles is found by applying the Nyquist criterion to $\mathfrak{D}_{2}(s)$ directly.

A few examples will serve to illustrate this technique. To discuss the stability of any bipropellant rocket, two sets of parameters must be known; $H, K$, and $n$ which depend upon the propellants and their mixture ratio; and $P_{0}, P_{f}, J_{0}, J_{f}, \alpha_{0}, \alpha_{f}$ which are associated with the propellant pumps, feed lines, and injector. In particular, the parameters $H, K$, and probably $n$ are not independent of each other; $H$ depends upon the propellant mixture ratio, and $K$ upon the slope of the combustion temperature vs. mixture ratio curve at the steady mixture ratio under consideration. For the examples to follow, these values will be taken as given in Table 1 for various mixture ratios.

\begin{tabular}{ccccl}
\multicolumn{5}{c}{ TABLE 1 } \\
$\bar{r}$ & $\bar{T}_{g}\left({ }^{\circ} \mathrm{F}\right)$ & $\frac{d \bar{T}_{g}}{d \bar{r}}$ & \multicolumn{1}{c}{$H$} & \multicolumn{1}{c}{$K$} \\
2.75 & 5065 & 0 & 0.2335 & 0 \\
2.50 & 5010 & 360 & 0.2125 & 0.09 \\
2.25 & 4880 & 640 & 0.1925 & 0.1475 \\
2.00 & 4706 & 850 & 0.167 & 0.189
\end{tabular}

For the first examples, choose the mechanical properties of the system such that 


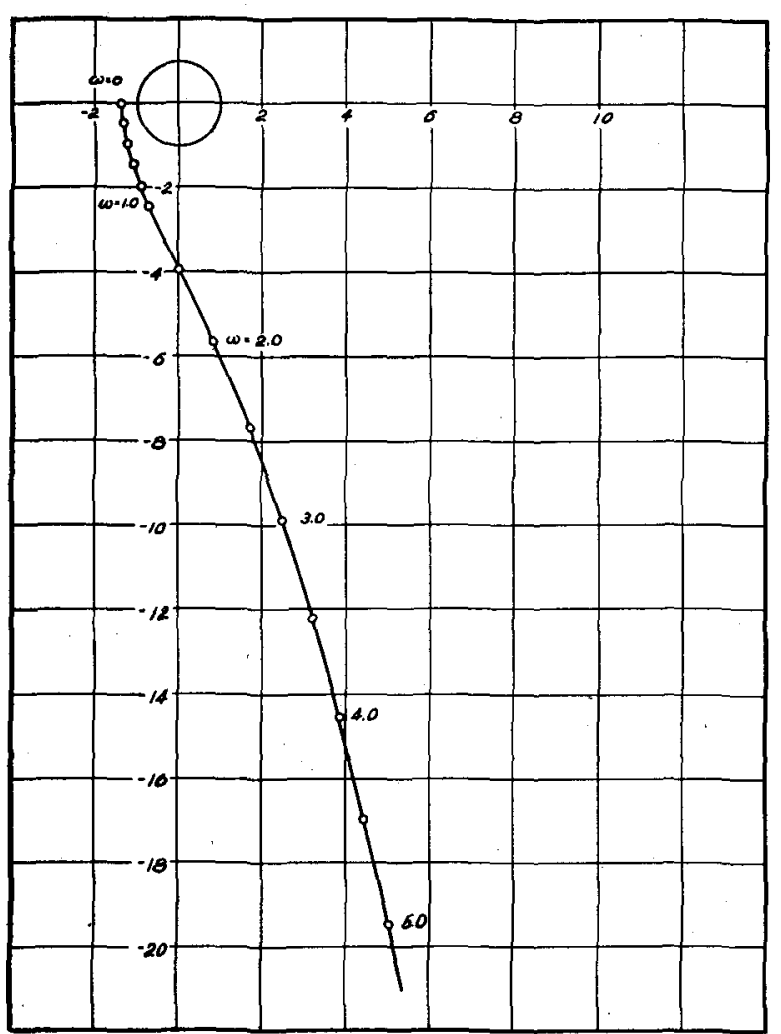

FIG. 3 SATCHE DIAGRAM FOR BIPROPELLANT ROCKET, $\bar{\gamma}=2.5$, $n=0.2 ; \alpha_{0}=\alpha_{f}=1.0, P_{0}=P_{f}=1.0, J_{0}=2.0, J_{f}=1.5$

$$
\begin{aligned}
& \alpha_{0}=\alpha_{f}=1.0 \\
& P_{0}=P_{f}=1.0 \\
& J_{0}=2.0 ; J_{f}=1.5
\end{aligned}
$$

and consider four cases corresponding to two values of the steady-state mixture ratio and two values of the coefficient $n=d \log f / d \log \bar{p}$.

$\begin{array}{ccc} & n & \bar{r} \\ 1 & 0.2 & 2.5 \\ 2 & 0.2 & 2.0 \\ 3 & 0.6 & 2.5 \\ 4 & 0.6 & 2.0\end{array}$

Then for calculating the functions $D_{1}(s)$ and $D_{2}(s)$ for values of $s$ on the imaginary axis, that is, for $s=i \omega$

$$
\begin{aligned}
\mathfrak{D}_{1}(i \omega)=\left\{\frac{25}{4}(1-n)-\left[\frac{35}{4}+\right.\right. & \left.3(1-n)] \omega^{2}\right\}+ \\
& i\left\{\left[\frac{25}{4}+\frac{35}{4}(1-n)\right] \omega-3 \omega^{3}\right\}
\end{aligned}
$$

$\mathfrak{D}_{2}(i \omega)=\left\{\frac{25}{4} n+\frac{5}{2}-3 n \omega^{2}\right\}+$

$$
i \omega\left\{\frac{35}{4} n-\frac{1}{2}(2 K+H)+\frac{7}{4}\right\}+\frac{1}{2} K\{i \omega \cos \omega+\omega \sin \omega\}
$$

The curves of $g_{2}(i \omega)$ for $n=0.2$, calculated using these relations, are shown in Fig. 3 for $\bar{\gamma}=2.5$, and in Fig. 4 for $\bar{\gamma}=$ 2.0. They are nearly identical despite the considerable difference in mixture ratio; neither of them intersect the unit circle. To close the diagram, take $s=R e^{i \vartheta}$ where $\vartheta$ decreases from $\pi / 2$ to $-(\pi / 2)$. For large values of $R, g_{2}(s)$ behaves as $-J_{0} J_{f} R^{3} e^{3 i \vartheta} / n J_{0} J_{f} R^{2} e^{2 i \vartheta}=-(R / n) e^{i \vartheta}$. Thus the $g_{2}(s)$ curve closes by a large arc in the left half of the Satche diagram, progressing clockwise. Since $g_{2}(s)$ neither crosses the unit circle nor encircles the origin outside the unit circle, instability can occur only if a zero of $D_{2}(s)$ exists which cancels a root of $g_{2}(s)$ in the right half $s$-plane. The presence of such a zero may be detected by applying the Nyquist criterion to $D_{2}(s)$. The Nyquist diagram is shown in Fig. 5 for $n=0.2$ and $\dot{r}=2.5$. For large values of $R, D_{2}\left(R e^{i \vartheta}\right)$ be-

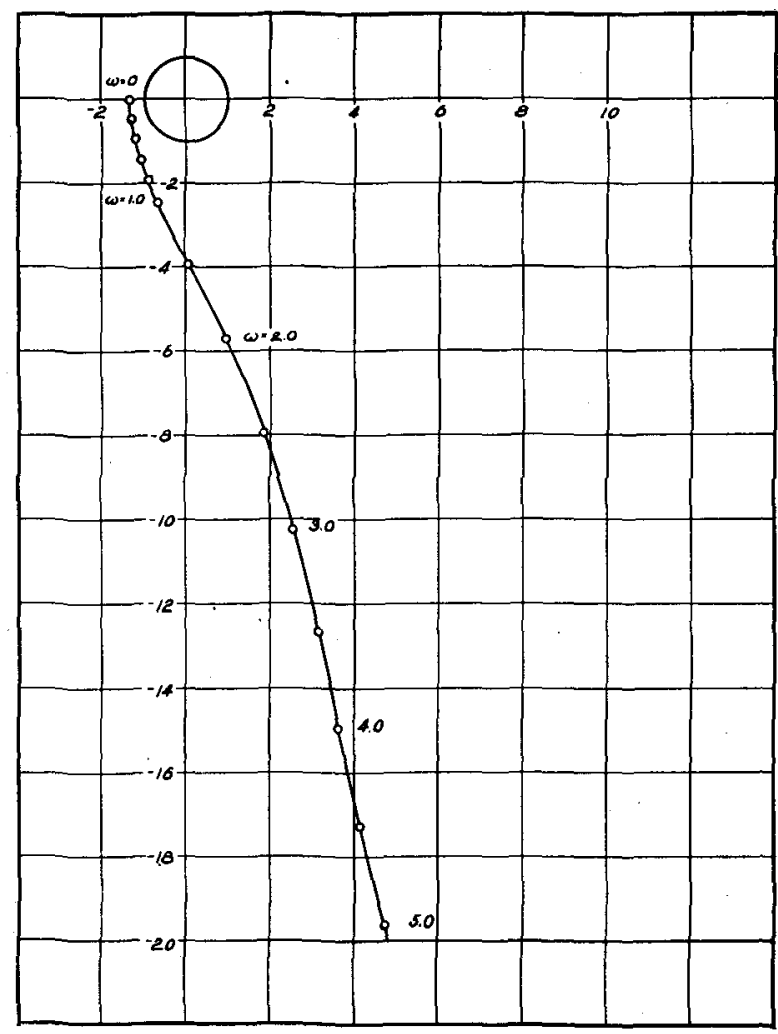

FIG. 4 SATCHE DIAGRAM FOR BIPROPELLANT ROCKET, $\bar{r}=2.0$, $n=0.2 ; \alpha_{0}=\alpha_{f}=1.0, P_{0}=P_{f}=1.0, J_{0}=2.0, J_{f}=1.5$

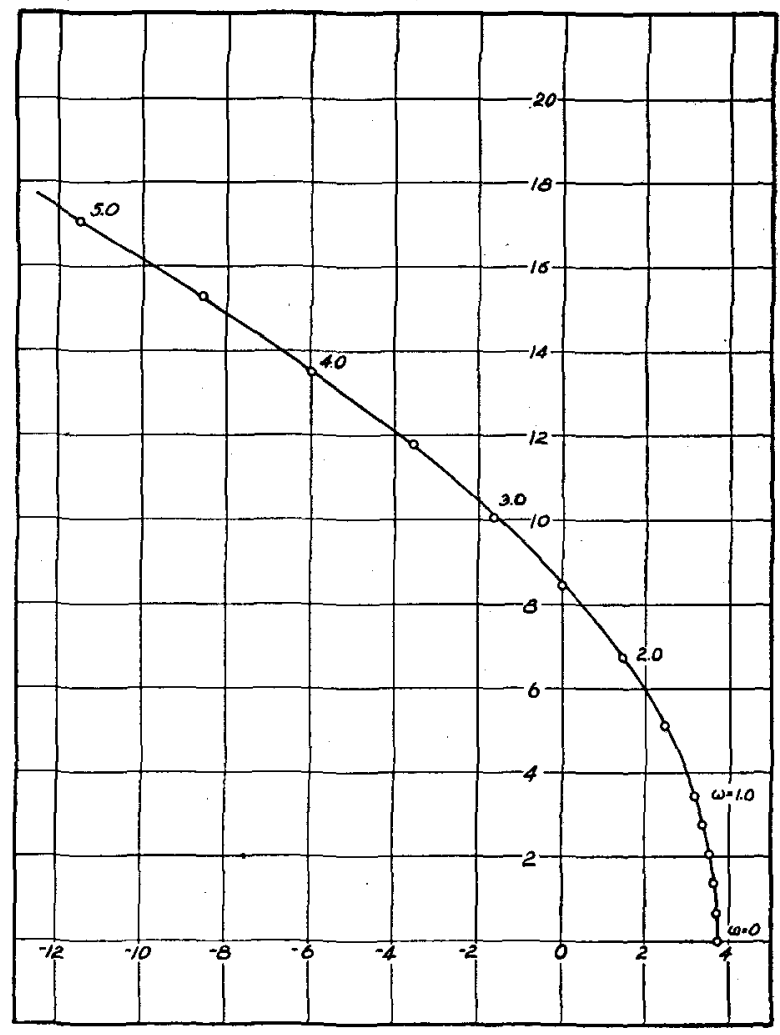

FIG. 5 NYQUIST DIAGRAM FOR $D_{2}(s), \bar{r}=2.5, n=0.2 ; \alpha_{0}=\alpha_{f}=$ 1.0, $P_{0}=P_{f}=1.0, J_{0}=2.0, J_{f}=1.5$

haves as $n J_{0} J_{f} R^{2} e^{2 i \vartheta}$, and therefore the Nyquist diagram closes with a large arc of $2 \pi$ radians starting from the upper branch in Fig. 5 and progressing clockwise to the corresponding lower branch (not shown). Since the origin is not enclosed, the system is certainly stable. The same conclusion may easily be reached for the lower mixture ratio, $\bar{\gamma}=2.0$, $n=0.2$. 


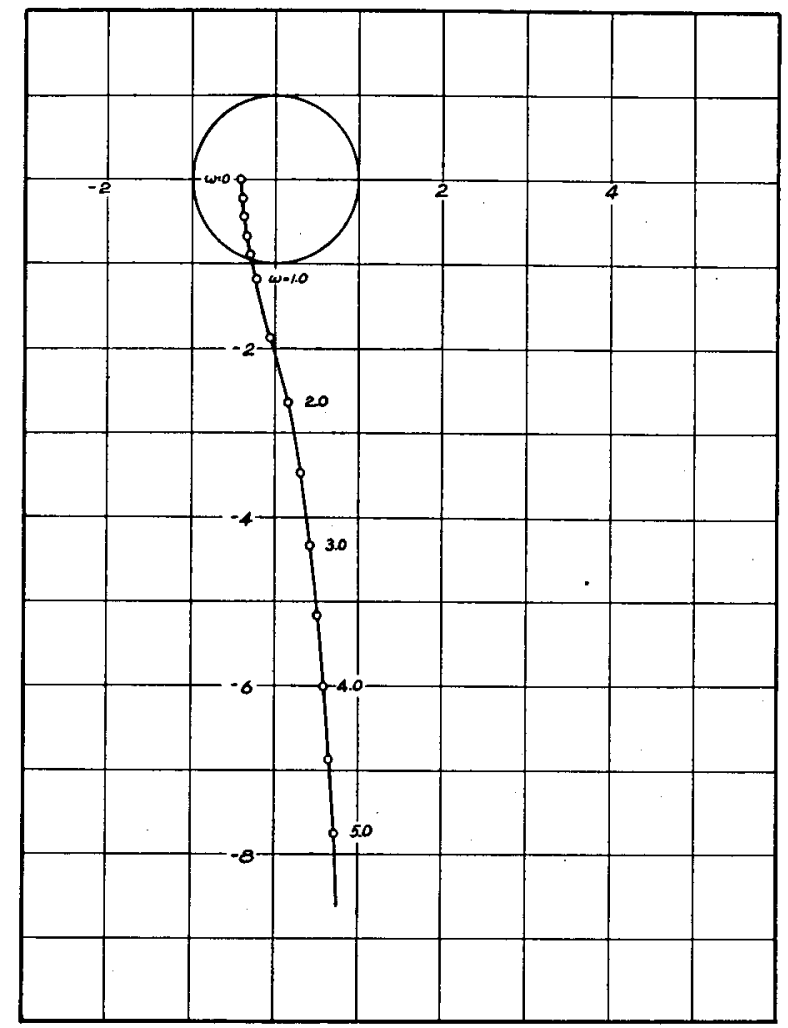

FIG. 6 SATCHE DIAGRAM FOR UNSTABLE BIPROPELLANT ROCKET, $\bar{r}=2.5, n=0.6 ; \alpha_{0}=\alpha_{f}=1.0, P_{0}=P_{f}=1.0, J_{0}=2.0$, $J_{f}=1.5$

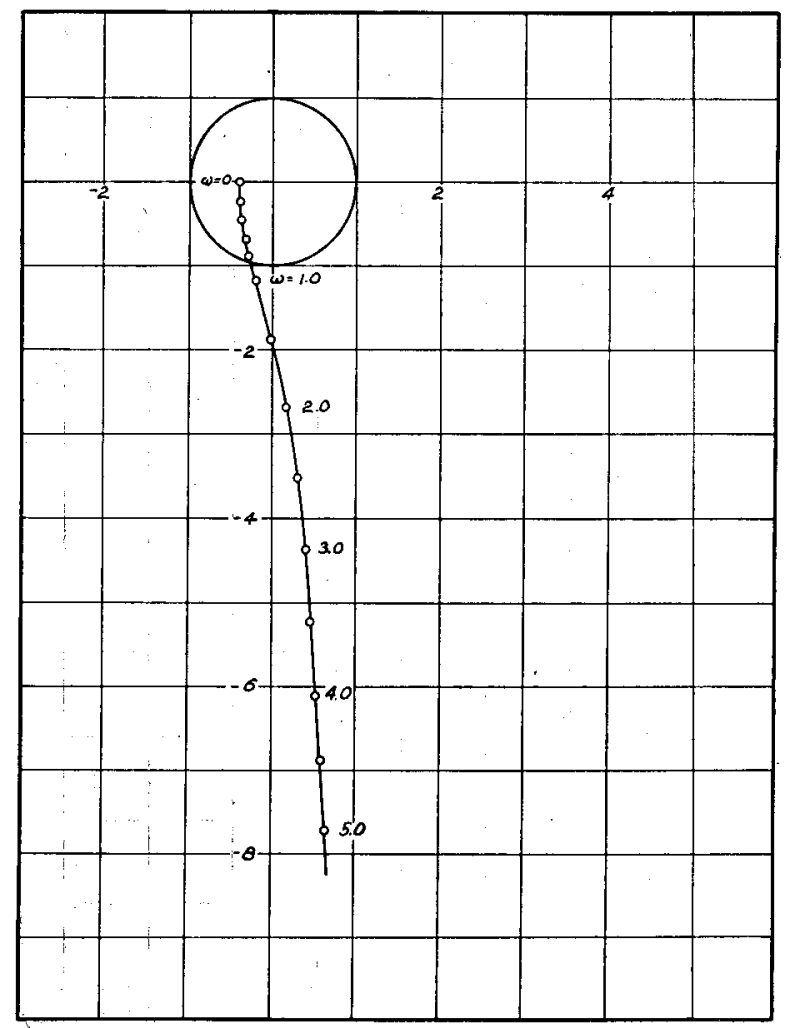

FIG. 7 SATCHE DIAGRAM FOR UNSTABLE BIPROPELLANT ROCKET, $\bar{r}=2.0, n=0.6 ; \alpha_{0}=\alpha_{f}=1.0, P_{0}=P_{f}=1.0, J_{0}=2.0$, $J_{f}=1.5$

The stability curves for the two corresponding examples with $n=0.6$, shown in Fig. 6 for $\bar{r}=2.5$, and in Fig. 7 for $\bar{r}=2.0$, both intersect the unit circle. Therefore a rocket propellant combination having parameters of these values will exhibit a low-frequency instability for some range of time lag $\delta$. The method for determining the critical time lag has

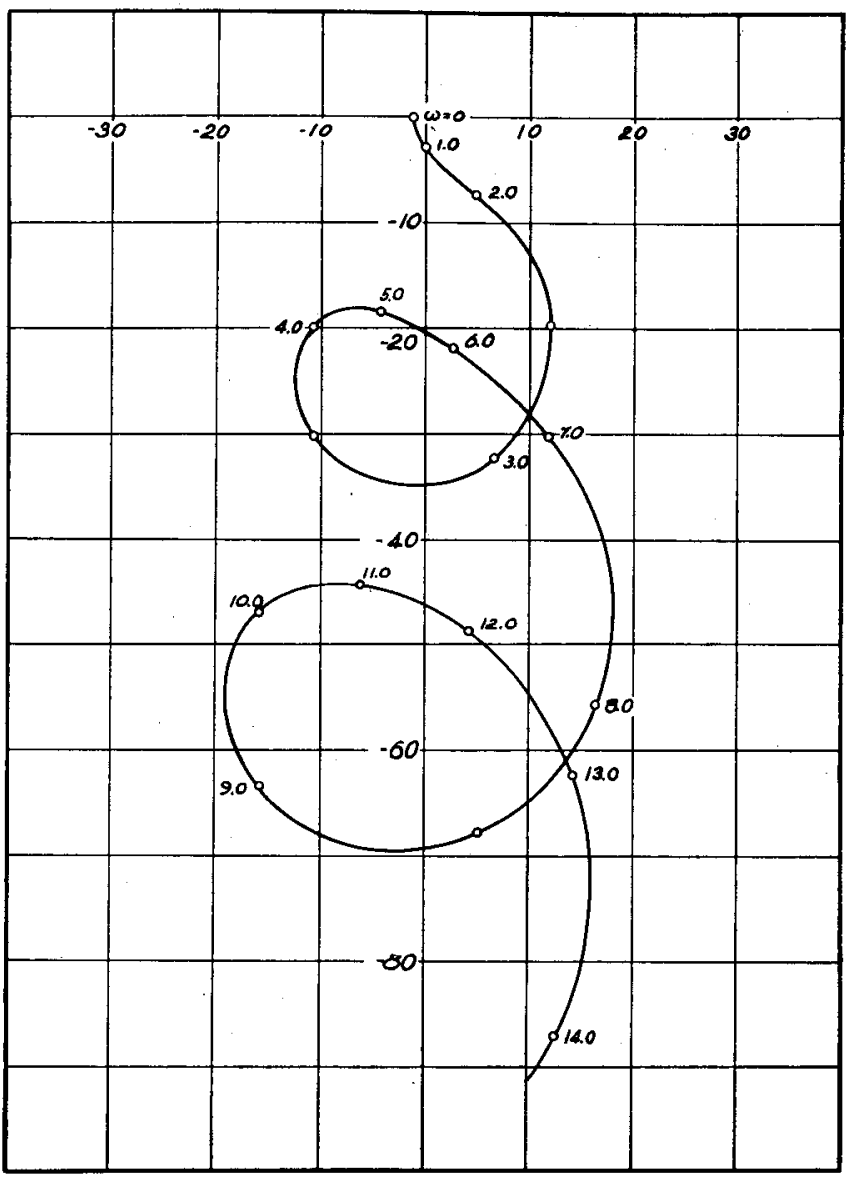

FIG. 8 SATCHE DIAGRAM FOR BIPROPELLANT ROCKET, $\bar{r}=2.0$ $n=0.2 ; \alpha_{0}=\alpha_{f}=1.0, P_{0}=1.0, P_{f}=4.0, J_{0}=4.0, J_{f}=1.0$

been shown by Tsien (5) in his original discussion of this method of analysis. Again the curves for the two values of mixture ratio are very similar. As in the first two examples, the closing arcs in the Satche diagram indicate no further instability of the system.

Because the mechanical properties of the two propellant systems are nearly identical in the foregoing examples, the change in mixture ratio associated with an oscillation in chamber pressure is very small and the behavior is much like that of a monopropellant rocket without line elasticity. Consequently the Satche diagrams are particularly simple. The influence of mixture ratio oscillation becomes important when the two lines have different inertial constants and injection pressures. As an example, consider a rocket for which

$$
\begin{array}{cl}
\alpha_{0}=\alpha_{f}=1.0 \\
J_{0}=4.0 & J_{f}=1.0 \\
P_{0}=1.0 & P_{f}=4.0 \\
\vec{r}=2.0 & n=0.2
\end{array}
$$

The equations for $\mathscr{D}_{1}(i \omega)$ and $\mathscr{D}_{2}(i \omega)$ may be written

$$
\begin{aligned}
& D_{1}(i \omega)=\left.\frac{55}{4}(1-n)-\left[\frac{49}{2}+4(1-n)\right] \omega^{2}\right\}+ \\
& i\left\{\left[\frac{55}{4}+\frac{49}{2}(1-n)\right] \omega-4 \omega^{2}\right\} \\
& D_{2}(i \omega)=\left\{\frac{55}{4} n+\frac{31}{4}-\frac{9}{2}(2 K-H)-4 n \omega^{2}\right\}+ \\
& i \omega\left\{\frac{49}{2} n+\frac{17}{2}-15(2 K+H)\right\}+ \\
& \frac{9}{2} K(\cos \omega-i \sin \omega)+15 K(i \omega \cos \omega+\omega \sin \omega)
\end{aligned}
$$

The Satche diagram for this example, Fig. 8, exhibits large loops. These loops originate in the trigonometric terms of $D_{2}(i \omega)$ which, in turn, arise because the change in combustion 
temperature associated with a change in mixture ratio does not affect the chamber discharge rate until the residence time $\vartheta_{\theta}$ later. Consequently these terms are associated with a reduced time lag of unity.

Physically the influence of these terms is easy to see. When a gas mass of low temperature, caused by a fluctuation in mixture ratio, flows to the chamber discharge nozzle, the mass flow rate increases (cf. Equation [8]), causing the chamber pressure to drop. This reduction of chamber pressure causes a variation of mixture ratio which may lead to another negative fluctuation in gas temperature. Clearly, at certain frequencies, these oscillations will reinforce each other so that they will, as in the case of the present calculation, be slowly damped. (As pointed out by Tsien, the Satche diagram indicates possible slowly damped oscillations when the $g_{2}(i \omega)$ curve passes close to the unit circle.) Furthermore, it is clear that there will be an infinite number of fre-

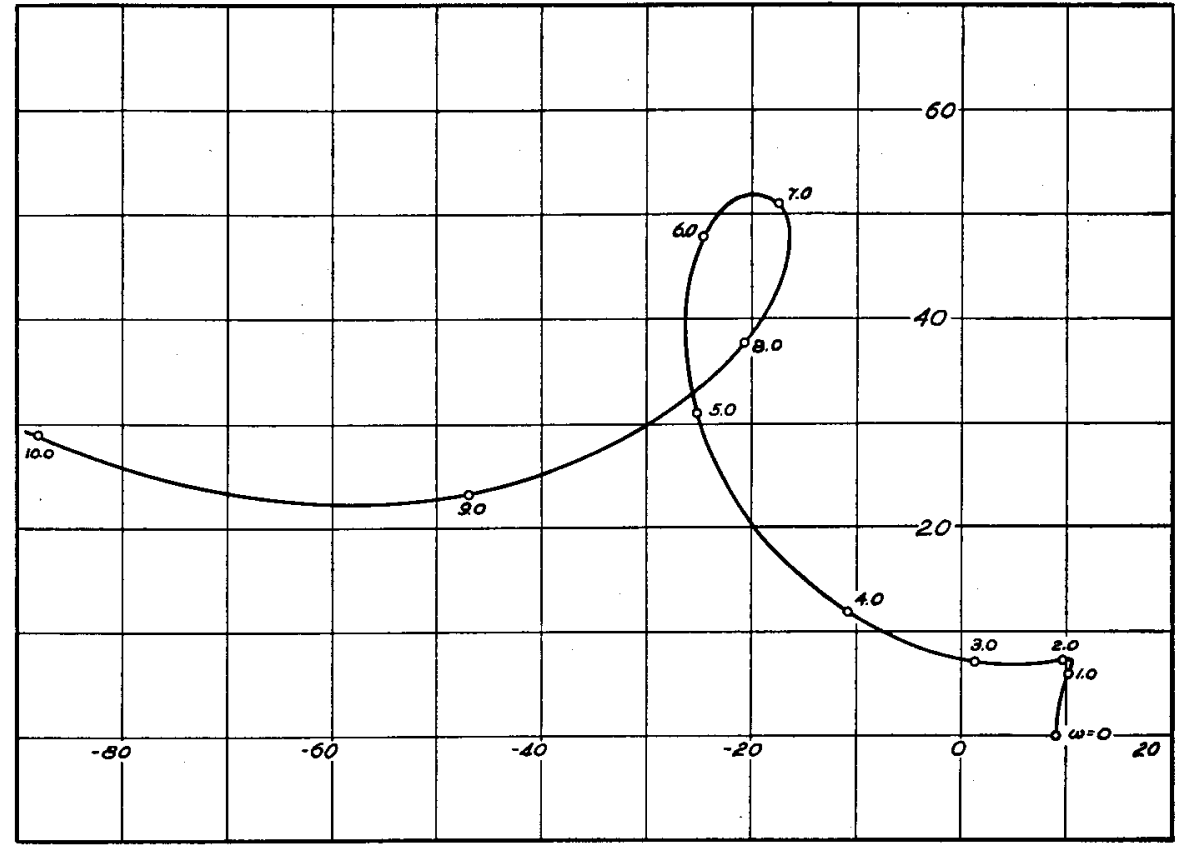

FIG. 9 NYQUIST DIAGRAM ASSOCIATED WITH SATCHE DIAGRAM OF FIG. 8 quencies at which such a reinforcement will take place. In the present example only two show up to any extent. The amplitude of an oscillation which originates in this manner depends to a considerable extent upon the response of the propellant feed system. If the change in pressure produces a large change in propellant flow, the oscillations may be severe; if the response is poor, the oscillation will disappear quickly. Now at high frequencies the inertia of the propellant supply causes the amplitude of its response to decrease, and consequently this particular resonance disappears. Mathematically this may be seen in the fact that for large values of $\omega$ the term $n J_{0} J_{f} \omega^{2}$ governs the behavior of $\mathscr{D}_{2}(i \omega)$, whereas

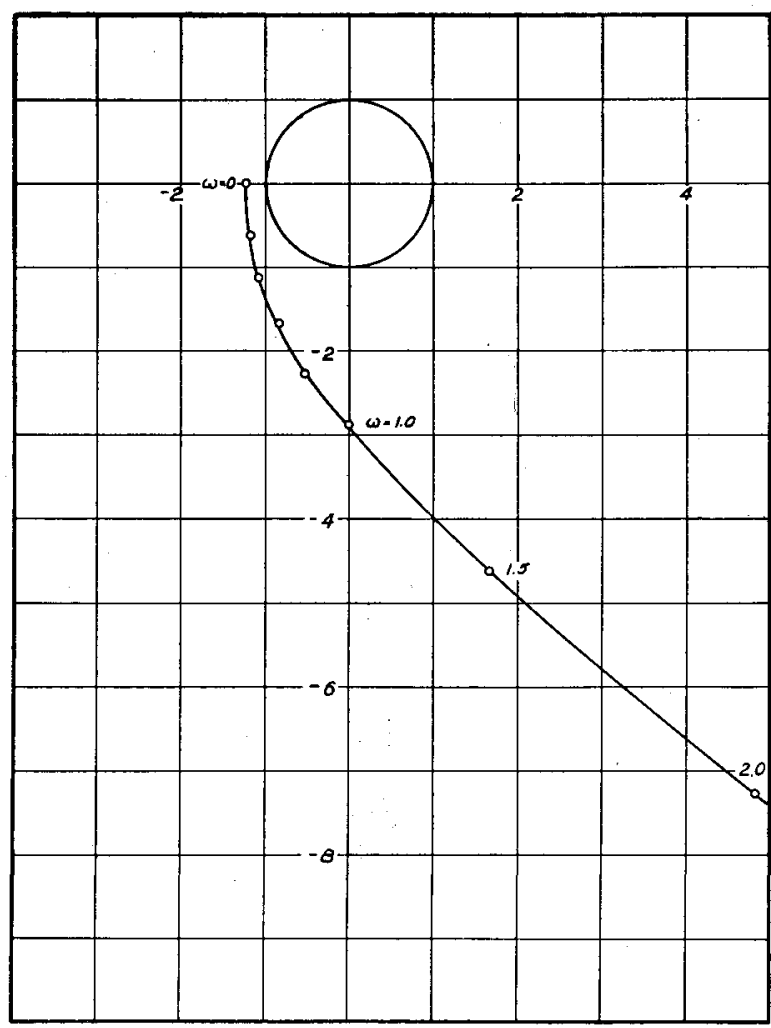

FIG. 10 DETAILS OF SATChE DIAGRaM NEAR UNIT CIRCLE, $\bar{r}=2.0, n=0.2 ; \alpha_{0}=\alpha_{j}=1.0, P_{0}=1.0, P_{f}=4.0, J_{0}=4.0$, $J_{f}=1.0$ the trigonometric terms are at most proportional to $\omega$.

The closing are of the $g_{2}(s)$ curve is the same as those discussed in previous examples. The Nyquist diagram of the denominator $\mathfrak{D}_{2}(s)$ is shown in Fig. 9, indicating no zeros of this determinant in the right half of the s-plane. The details of the Satche diagram near the unit circle, Fig. 10, show that the curve lies outside the unit circle and, as a consequence of this and the above considerations, the system is conclusively stable for all values of the combustion time lag.

The influence of mixture ratio variation may be further increased by considering a propellant combination for which the curve of combustion temperature vs. mixture ratio has a

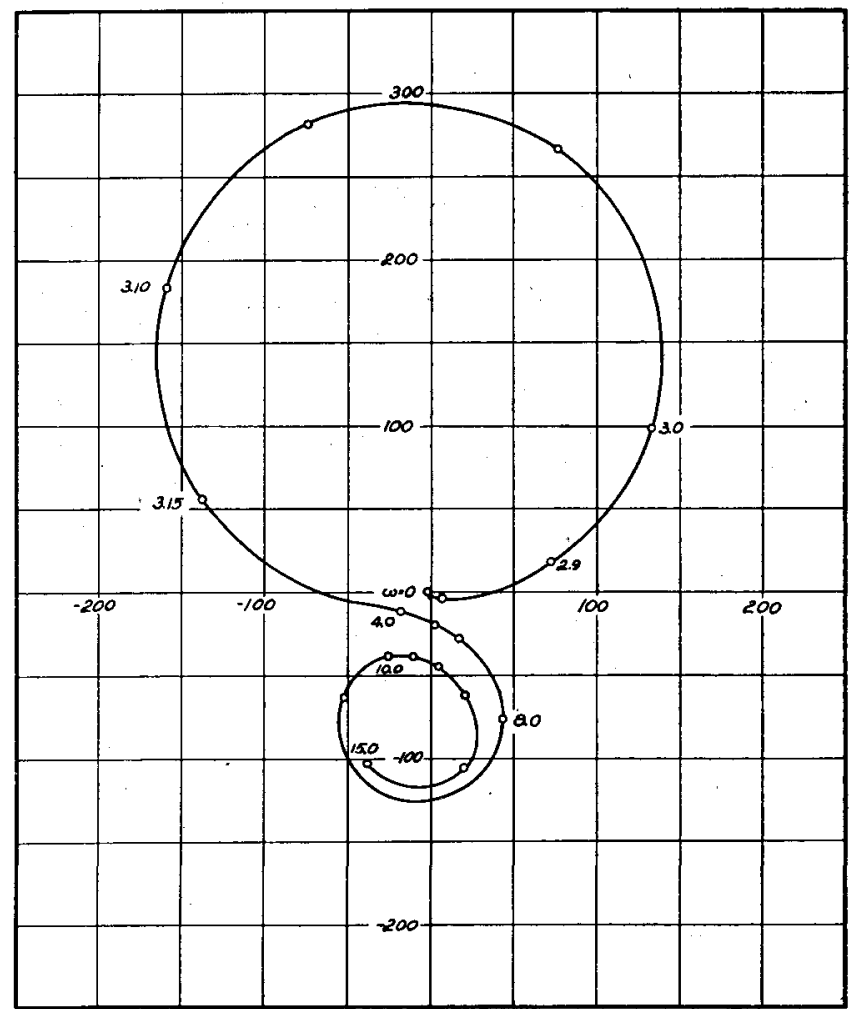

FIG. 11 SATCHE DIAGRAM FOR BIPROPELLANT ROCKET, $K=\mathbf{0 . 3}$, $\bar{r}=2.0, n=0.2 ; \alpha_{0}=\alpha_{f}=1.0, P_{0}=1.0, P_{f}=4.0, J_{0}=4.0$, $J_{f}=1.0$ 
slope greater than that considered in the previous example. Let all values of the previous example be retained with the exception that $K$ is arbitrarily increased from 0.189 to 0.30 . The resulting Satche diagram, Fig. 11, shows that the loops have now grown so large as to encircle the origin. By drawing the closing are and checking the course of the complete curve, it is simply found that the $g_{2}(s)$ curve encircles the origin twice in a counterclockwise direction. The Nyquist diagram of $D_{2}(s)$, Fig. 12, shows a corresponding double encirclement but in the clockwise direction. Consequently, this cancellation indicates that no instability can arise from encirclement of the origin in the Satche diagram. Furthermore, the details of the Satche diagram in the neighborhood of the unit circle, Fig. 13, show no intersection and, thus, the system is definitely stable against all values of the combustion time lag.

\section{Servo-Stabilization of the Bipropellant Rocket}

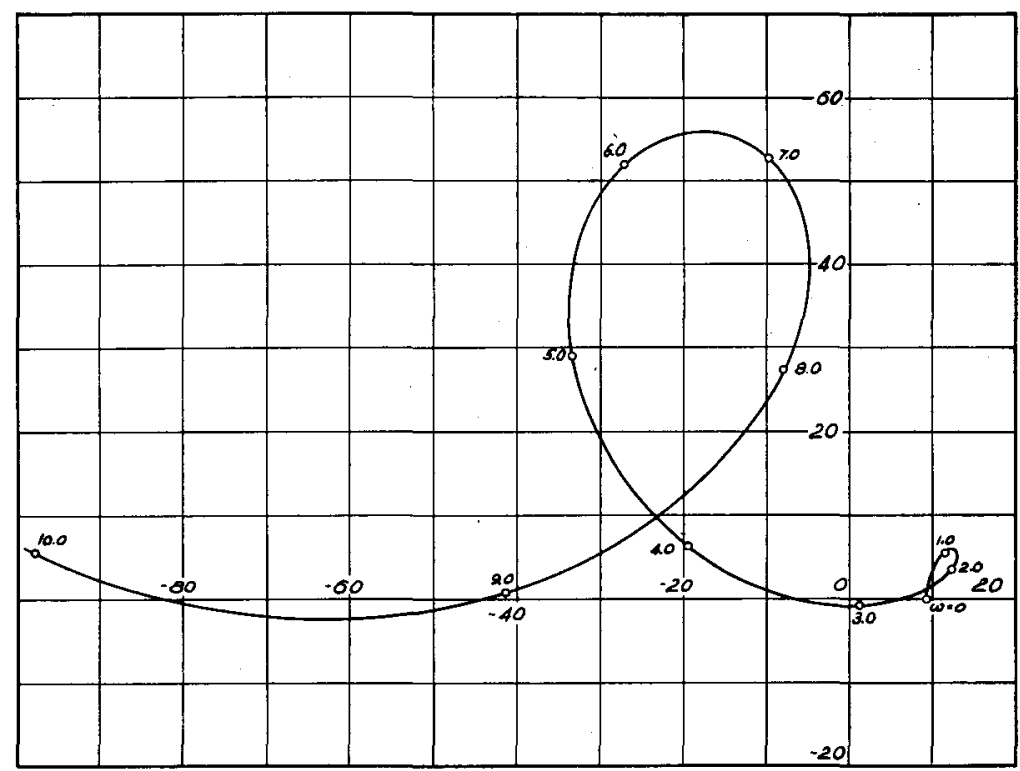

FIG. 12 NYQUIST DIAGRAM ASSOCIATED WITH SATCHE DIAGRAM OF FIG. 11

Where possible unstable operation of a rocket is indicated, stability may often be assured by modifying the mechanical design of the propellant supply system or injector, or in the extreme, by change in the chemical propellants. Alternatively the stability may be assured by introducing a feedback loop connecting the combustion chamber and the variable capacitances in each propellant line. In the simplest case the loop will consist of a pressure measuring device attached to the rocket chamber, an amplifier, and an appropriate servomechanism to actuate the capacitances in the propellant lines. For the bipropellant rocket, Fig. 14, two separate circuits may be employed, one for each propellant line. However, in the interest of simplicity it may be preferable (Fig. 15) to control only a single propellant line, or to control both through a single amplifier, building any required differences into the servomechanisms or into the capacitances. ${ }^{4}$

In general, then, the capacitances $\kappa_{0}$ and $\kappa_{f}$ will be made to vary with time in a manner prescribed according to the variation in chamber pressure. Employing dimensionless forms, the correspondences between the capacitances and the chamber pressure may be written symbolically

$$
\begin{aligned}
& \kappa_{0}(z)=F_{0}\left(\frac{d}{d z}\right) \varphi(z) . \\
& \kappa_{f}(z)=F_{f}\left(\frac{d}{d z}\right) \varphi(z) .
\end{aligned}
$$

where $F_{0}(d / d z)$ and $F_{f}(d / d z)$ are linear differential-integral operators. These relations simply indicate that the two capacitances are related to the chamber pressure through linear differential equations with constant coefficients, the forms of which depend upon the pressure pickup, the amplifiers, and the servomechanisms.

The problem is then to design the feedback circuits, that is, to determine the forms of $F_{0}(d / d z)$ and $F_{f}(d / d z)$ such that stable rocket operation is achieved. This resolves, practically, to the design of amplifiers to work with given servomechanisms and with a given pressure pickup so as to produce the required forms of $F_{0}$ and $F_{f}$. The proper forms of these functions can be determined only through stability analysis of the complete set of five differential equations, the original set consisting of Equation [11], and two of the type of Equation [13] augmented by the two equations arising from

4 It has come to the attention of the authors that Y. C. Lee, M. R. Gore, and C. C. Ross, of the Aerojet Engineering Corporation, have considered a somewhat different arrangement in their paper, "Stability and Control of Liquid Propellant Rocket Systems" (see pp. 75-81 in this issue of the JournaL).

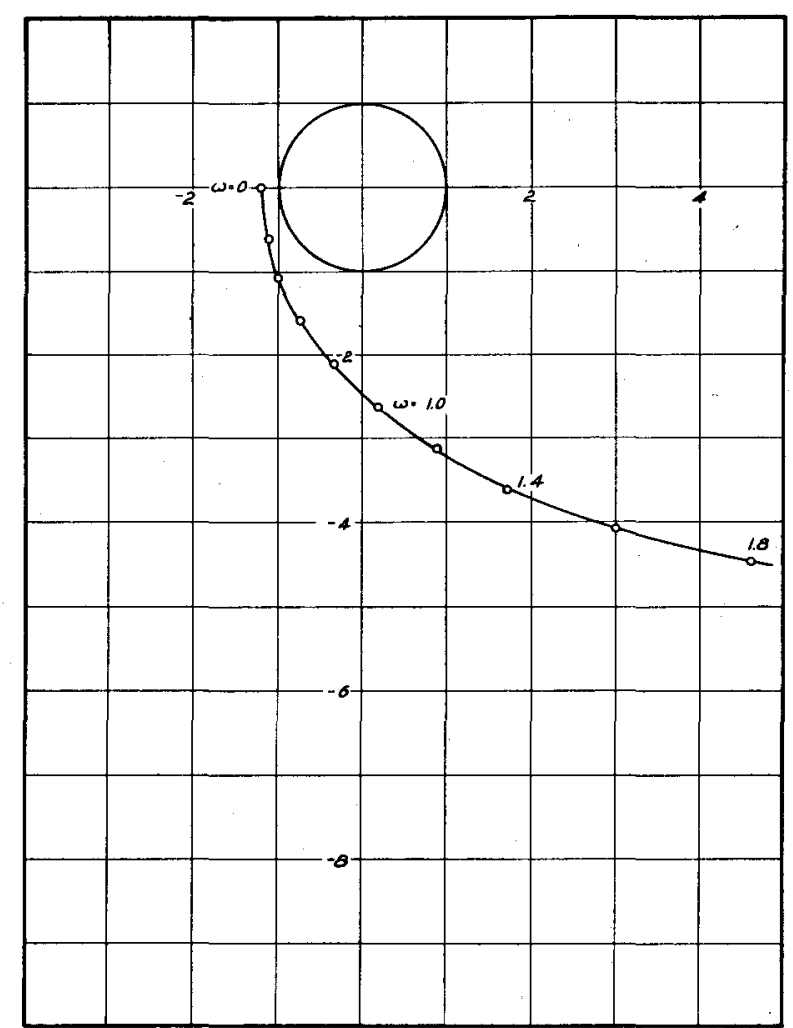

Fig. 13 DETAILS OF SATCHE DIAGRAM NEAR UNIT CIRCLE, $K=0.3, \bar{r}=2.0, n=0.2 ; \alpha_{0}=\alpha_{f}=1.0, P_{0}=1.0, P_{f}=4.0$, $J_{0}=4.0, J_{j}=1.0$

the feedback loops, Equations [23] and [24]. Two additional dependent variables $\kappa_{0}(z)$ and $\kappa_{f}(z)$ have been introduced by allowing the capacitances to vary. The Laplace transforms of these equations are given in Appendix 1, and retaining only the homogeneous portion for stability analysis, the set of algebraic equations is

$$
\begin{aligned}
& {\left[s+1-n+n e^{-s \delta}\right] \Phi(s)+} \\
& e^{-s \delta}\left[K e^{-s}-\left(2 K+H+\frac{1}{2}\right)\right] M_{0}(s)- \\
& \quad e^{-s \delta}\left[K e^{-s}-\left(2 K+H-\frac{1}{2}\right)\right] M_{f}(s)=0
\end{aligned}
$$




$$
\begin{aligned}
P_{0} \Phi(s)+\left[J_{0 s}+1+\frac{1}{\alpha_{0}}(\right. & \left.\left.P_{0}+\frac{1}{2}\right)\right] M_{0}(s)+ \\
& {\left[J_{0} s^{2}+\frac{1}{\alpha_{0}}\left(P_{0}+\frac{1}{2}\right) s\right] K_{0}(s)=0 }
\end{aligned}
$$$$
P_{f} \Phi(s)+\left[J_{f} s+1+\frac{1}{\alpha_{f}}\left(P_{f}+\frac{1}{2}\right)\right] M_{f}(s)+
$$$$
\left[J_{f} s^{2}+\frac{1}{\alpha_{f}}\left(P_{f}+\frac{1}{2}\right) s\right] K_{f}(s)=0
$$$$
F_{0}(s) \Phi(s)-K_{0}(s)=0
$$$$
F_{f}(s) \Phi(s)-K_{f}(s)=0 .
$$

where $K_{0}(s)$ and $K_{f}(s)$ are the Laplace transforms of the variable capacitances; $F_{0}(s)$ and $F_{f}(s)$ are the transfer functions of the feedback loops. The stability of the system is now dependent upon the roots of the fifth-order determinant constructed from the coefficients of Equations [25]

$$
\begin{aligned}
& \left.\mid \begin{array}{ccc}
s+1-n+n e^{-s \delta} & e^{-s \delta}\left[K e^{-s}-\right. & -e^{-s \delta}\left[K e^{-s}-\right. \\
& \left.\left(2 K+H+\frac{1}{2}\right)\right] & \left(2 K+H-\frac{1}{2}\right)
\end{array}\right] \\
& \begin{aligned}
& P_{0} J_{0} s+\frac{1}{1}+ \\
& \frac{1}{\alpha_{0}}\left(P_{0}+\frac{1}{2}\right)
\end{aligned} \\
& P_{f} \quad 0 \\
& \begin{array}{ll}
F_{0}(s) & 0 \\
F_{f}(s) & 0
\end{array} \\
& \begin{aligned}
& J_{f} s+ 1+ \\
& \frac{1}{\alpha_{f}}\left(P_{f}+\frac{1}{2}\right)
\end{aligned} \\
& 0 \\
& 0 \\
& D_{0}(s)= \\
& \begin{array}{ll}
K e^{-s}- & -\left[K e^{-s}-\right. \\
\left(2 K+H+\frac{1}{2}\right) & \left.\left(2 K+H-\frac{1}{2}\right)\right]
\end{array} \\
& J_{0} s+1+ \\
& \begin{array}{ccc}
\frac{1}{\alpha_{0}}\left(P_{0}+\frac{1}{2}\right) & 0 & J_{0} s^{2}+ \\
0 & J_{f} s+\frac{1}{\alpha_{0}}\left(P_{0}+\frac{1}{2}\right) s \\
\frac{1}{\alpha_{f}}\left(P_{f}+\frac{1}{2}\right) & 0
\end{array}
\end{aligned}
$$

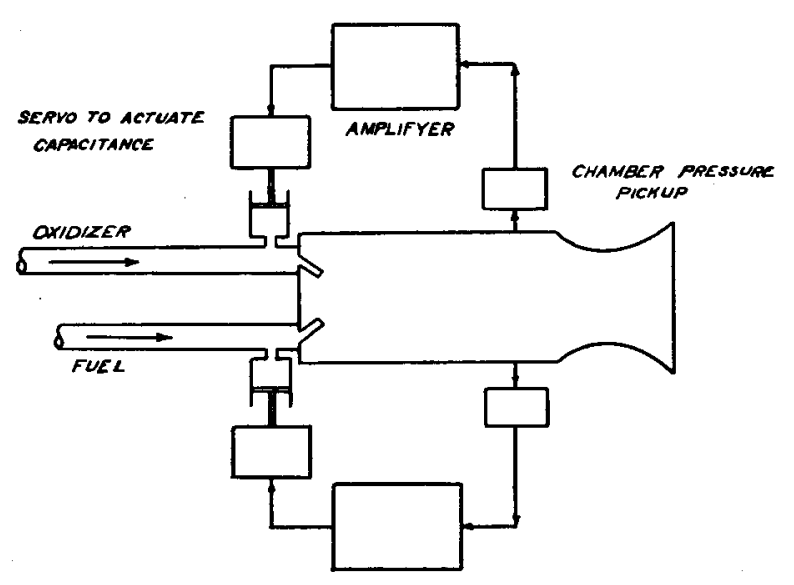

FIG. 14 SCHEMATIC DIAGRAM OF BIPROPELLANT ROCKET INCORPORATING FEEDBACK SYSTEM

$J_{0} s^{2}+\frac{1}{\alpha_{0}}\left(P_{0}+\frac{1}{2}\right) s \quad 0$

0

$J_{f} s^{2}+\frac{1}{\alpha_{f}}\left(P_{f}+\frac{1}{2}\right) s$

$-1$

0

$-1$$$
\mathfrak{D}_{f}(s)=
$$

$$
\left|\begin{array}{ccc}
K e-s- & -[K e-s- & \\
\left(2 K+H-\frac{1}{2}\right) & \left.\left(2 K+\mathrm{H}+\frac{1}{2}\right)\right] & 0 \\
J_{f} s+\frac{1}{1}+ & 0 & J_{f} s^{2}+ \\
\frac{1}{\alpha_{f}}\left(P_{f}+\frac{1}{2}\right) & \frac{1}{\alpha_{f}}\left(P_{f}+\frac{1}{2}\right) s \\
0 & J_{0 s}+\frac{1}{\alpha_{0}}\left(P_{0}+\frac{1}{2}\right) & 0
\end{array}\right|
$$

The characteristic equation obtained by setting the stability determinant (Equation [26]) to zero is

$$
\mathfrak{D}_{1}(s)+e^{-s \delta}\left[\mathfrak{D}_{2}(s)+F_{0}(s) \mathfrak{D}_{0}(s)+F_{f}(s) \mathfrak{D}_{f}(s)\right]=0 \ldots[29]
$$

The two transfer functions of the feedback loops, $F_{0}(s)$ and
$F_{f}(s)$, appear as multiplicative factors; each of the determinants depends upon only the original rocket configuration and is independent of the feedback circuit.

The new form of the function $g_{2}(s)$ is
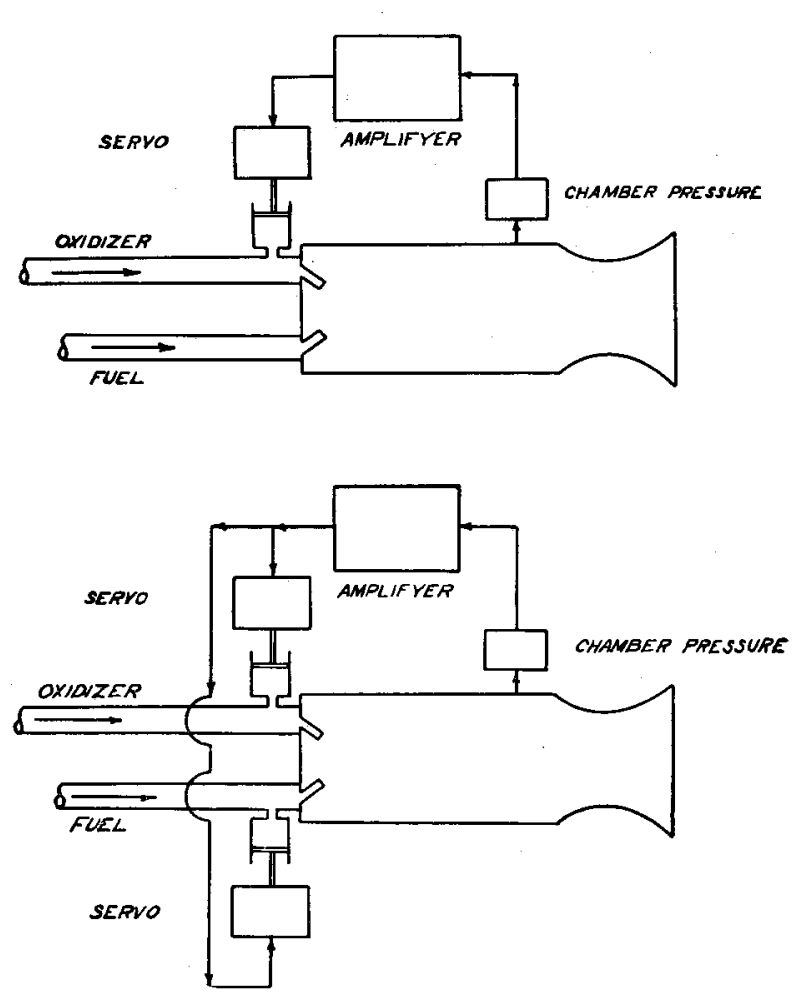

FIG. 15 TWO FEEDBACK CIRCUITS EMPLOYNG ONLY A SINGLE AMPLIFIER AND PICKUP 


$$
g_{2} s=\frac{D_{1}(s)}{\mathscr{D}_{2}(s)+F_{0}(s) D_{0}(s)+F_{f}(s) D_{f}(s)}
$$

Stable rocket operation is to be assured by choosing realizable forms of the feedback transfer functions $F_{0}(s)$ and $F_{f}(s)$ so that the Satche diagram satisfies the criteria previously discussed. One convenient procedure is to choose an appropriate Satche diagram and solve for the feedback transfer functions. For the present system the result is usually not a realizable or convenient feedback circuit.

When the instability arises from intersection of the $g_{2}(i \omega)$ curve with the unit circle, as it appears to do in practical cases of low-frequency oscillation, a proper choice of $F_{0}(s)$ and $F_{f}(s)$ may be effected quite simply. The intersection, when it exists, occurs for value of $\omega$ considerably less than unity; if this were not true, the oscillations would be of rather high frequency considering residence times of the usual magnitude. Hence the modification of the $g_{2}(i \omega)$ function may be restricted to low values of $\omega$, this is to say, the gain of the amplifier in the feedback system should drop to a low value as the frequency $\omega$ increases. The appropriate analytic procedure is then $(a)$ to find the approximate behavior of $F_{0}(s)$ and $F_{f}(s)$ for $s=i \omega<<i$ such that $g_{2}(i \omega)$ does not intersect the unit circle, and $(b)$ to determine the functions $F_{0}(s)$ and $F_{f}(s)$ so that they have the prescribed behavior for small values of $\omega$ and vanish for large $\omega$, thus preserving the original Satche diagram in this range.

This program may be carried out in the following manner. Each of the third-order determinants involved in Equation $[30]$ is regular at $s=0$, and hence may be approximated by a power series

$$
\begin{aligned}
& D_{1}(s)=d_{1}{ }^{(0)}+d_{1}{ }^{(1)} s+d_{1}{ }^{(2)} s^{2}+d_{1}{ }^{(3)} s^{3} \\
& D_{2}(s)=d_{2}^{(0)}+d_{2}^{(1)} s+d_{2}^{(2)} s^{2}+d_{2}^{(3)} s^{3}+\ldots \\
& D_{0}(s)=d_{0}{ }^{(0)}+d_{0}{ }^{(1)} s+d_{0}{ }^{(2)} s^{2}+d_{0}{ }^{(3)} s^{3}+d_{0}{ }^{(4)} s^{4}+\ldots \\
& D_{f}(s)=d_{f}^{(0)}+d_{f}^{(1)} s+d_{f}^{(2)} s^{2}+d_{f}^{(3)} s^{3}+d_{f}^{(4)} s^{4}+\ldots
\end{aligned}
$$

The coefficients $d_{i}{ }^{(j)}$ depend only upon the parameters of the original (unstabilized) rocket and hence are known numerical values for any particular case. The expressions for these coefficients are given in Appendix 2. In particular, it appears that $d_{0}{ }^{(0)}=d_{f}{ }^{(0)}=0$, a result which is clear from inspection of $D_{0}(s)$ and $D_{f}(s)$ as given by Equations [27] and [28]. The transfer functions for the feedback loops jikewise possess power-series expansions about $s=0$, although they need not be regular at that point. From the fact that the determinants $\mathscr{D}_{0}(s)$ and $\mathscr{D}_{f}(s)$ behave as $s$ for small values of $s$, the expansions of the feedback transfer functions must behave as $1 / s$ in order that the value of $g_{2}(0)$ be changed from that of the original system. That is, the transfer function must correspond to a simple integrating circuit. Thus it is appropriate to choose

$$
\begin{aligned}
& F_{0}(s)=f_{0}^{(-1)} \frac{1}{s}+f_{0}^{(0)}+f_{0}^{(1)} s+f_{0}^{(2)} s^{2}+\ldots \\
& F_{f}(s)=f_{f}^{(-1)} \frac{1}{s}+f_{f}^{(0)}+f_{f}^{(1)} s+f_{f}^{(2)} s^{2}+\ldots
\end{aligned}
$$

where the $f_{0}{ }^{(j)}$ and the $f_{f}^{(j)}$ are unknown constants. Clearly, upon substituting the approximations of Equations [31] and [32] into Equation [30], a power-series representation for $g_{2}(s)$ is obtained where the unknown coefficients $f_{0}^{(j)}, f_{f}^{(j)}$ appear in the coefficients of the expansion.

From the viewpoint of insuring against low-frequency instability, the Satche diagram must be such that the $g_{2}(i \omega)$ curve lies outside the unit circle. For small $\omega$, which are involved near the unit circle, $g_{2}(i \omega)$ may be approximated by the polynomial

$$
g_{2}(i \omega)=\gamma_{0}+\gamma_{1}(i \omega)+\gamma_{2}(i \omega)^{2}+\gamma_{3}(i \omega)^{3}
$$

where the $\gamma_{i}$ are constants chosen so that this condition is satisfied. Then there are two expressions for $g_{2}(s)$, valid for small $s$

$$
g_{2}(s)=\gamma_{0}+\gamma_{1} s+\gamma_{2} s^{2}+\gamma_{3} s^{3} \ldots \ldots \ldots \ldots[33]
$$

and that which followed from expansion of Equation [30]. Since they are both valid power-series expansions, they are equal term by term. The result is four algebraic equations relating the constants $f_{0}^{(j)}$ and $f_{f}^{(j)}$ to the rocket parameters and to the chosen form of the Satche diagram near the unit circle. After some simplification these relations are

$$
\begin{aligned}
& d_{0}^{(1)} f_{0}{ }^{(-1)}+d_{f}^{(1)} f_{f}(-1)+d_{2}{ }^{(0)}=-\frac{d_{1}^{(0)}}{\gamma_{0}} \ldots \ldots \\
& d_{0}^{(2)} f_{0}{ }^{(-1)}+d_{0}{ }^{(1)} f_{0}{ }^{(0)}+d_{f}^{(2)} f_{f}^{(-1)}+d_{f}^{(1)} f_{f}^{(0)}+d_{2}^{(1)}= \\
& \frac{d_{1}{ }^{(0)}}{\gamma_{0}}\left(\frac{\gamma_{1}}{\gamma_{0}}-\frac{d_{1}^{(1)}}{d_{1}{ }^{(0)}}\right) \\
& d_{0}^{(3)} f_{0}{ }^{(-1)}+d_{0}^{(2)} f_{0}^{(0)}+d_{0}^{(1)} f_{0}^{(1)}+d_{f^{(3)}} f_{f}^{(-1)}+d_{f}^{(2)} f_{f}^{(0)}+ \\
& d_{f}^{(1)} f_{f}^{(1)}+d_{2}^{(2)}=\frac{d_{1}{ }^{(0)}}{\gamma_{0}}\left[\left(\frac{\gamma_{2}}{\gamma_{0}}-\frac{d_{1}^{(2)}}{d_{1}{ }^{(0)}}\right)-\frac{\gamma_{1}}{\gamma_{0}}\left(\frac{\gamma_{1}}{\gamma_{0}}-\frac{d_{1}{ }^{(1)}}{d_{1}{ }^{(0)}}\right)\right] \\
& d_{0}{ }^{(4)} f_{0}{ }^{(-1)}+d_{0}^{\left({ }^{(3)}\right.} f_{0}{ }^{(0)}+d_{0}^{(2)} f_{0}{ }^{(1)}+d_{0}{ }^{(1)} f_{0}{ }^{(2)}+d_{f}^{(4)} f_{f}^{(-1)}+
\end{aligned}
$$

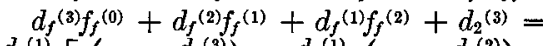

$$
\begin{aligned}
& \frac{d_{1}{ }^{(1)}}{\gamma_{0}}\left[\left(\frac{\gamma_{3}}{\gamma_{0}}-\frac{d_{1}{ }^{(3)}}{d_{1}{ }^{(0)}}\right)-\frac{d_{1}{ }^{(1)}}{d_{1}{ }^{(0)}}\left(\frac{\gamma_{2}}{\gamma_{0}}-\frac{d_{1}{ }^{(2)}}{d_{1}{ }^{(0)}}\right)-\right. \\
& \left.2\left(\frac{\gamma_{2}}{\gamma_{0}}-\frac{d_{1}^{(2)}}{d_{1}{ }^{(0)}}\right)\left(\frac{\gamma_{1}}{\gamma_{0}}-\frac{d_{1}^{(1)}}{d_{1}{ }^{(0)}}\right)+\left(\frac{\gamma_{1}^{2}}{\gamma_{0}{ }^{2}}-\frac{d_{1}{ }^{(2)}}{d_{1}{ }^{(0)}}\right)\left(\frac{\gamma_{1}}{\gamma_{0}}-\frac{d_{1}^{(1)}}{d_{1}{ }^{(0)}}\right)\right]
\end{aligned}
$$

Four more relations among the $f_{0}^{(j)}$ and $f_{f}^{(j)}$ may be chosen arbitrarily so as to fix their values uniquely. Two possibilities have been mentioned previously: (a) the feedback loop operates only on a single capacitance so that either $f_{0}^{(j)}=0$ or $f_{f}^{(j)}=0$; $(b)$ the two feedback loops are identical so that $f_{0}{ }^{(j)}=f_{f}{ }^{(j)} ; j=-1,0,1,2$. In each case only one pressuresensing device and amplifier are required.

With the values of $f_{0}^{(j)}, f_{f}^{(j)}$ known, the behavior of the feedback circuit is determined for small $s$; the task is now to obtain general representations for $F_{0}(s)$ and $F_{f}(s)$ which agree with the known expressions for small $s$ and vanish for large $s$ so as to leave the original Satche diagram unchanged for large s. If, for instance, it were decided to control the oxidizer flow and leave the fuel flow uncontrolled, $f_{f}{ }^{(j)}=0$ and the $f_{0}{ }^{(j)}$ are determined by successive application of Equations [34], [35], [36], and [37]. Then choose

$$
F_{0}(s)=\frac{f_{0}(-1)}{s}\left[\frac{1-c s}{(1-a s)\left(1-b s^{2}\right)}\right] \ldots \ldots \ldots
$$

It is clear by reference to Equation [32] that the singular behavior as $s \rightarrow 0$ is correct. Furthermore for large $s, F_{0}(s)$ vanishes as $1 / s^{3}$ which is probably strong enough. If the constants $a, b$, and $c$ can be found so that the expansion of Equation [38] yields the first few coefficients in agreement with the known values $f_{0}{ }^{(0)}, f_{0}{ }^{(1)}$, and $f_{0}{ }^{(2)}$, then Equation [36] will constitute a transfer function of the feedback loop, satisfactory for stabilizing the rocket against low-frequency oscillations regardless of the propellant time lag.

The power-series expansion of Equation [38] is

$$
\begin{aligned}
F_{0}(s)=\frac{f_{0}^{(-1)}}{s}\{1+(a-c) s+ & {[a(a-c)+b] s^{2}+} \\
& \left.\left(a^{2}-b\right)(a-c) s^{3}+\ldots\right\} \ldots[39]
\end{aligned}
$$

which must be equal, term by term, to the first of Equations [32]. This condition determines the constants $a, b, c$ directly as

$$
\begin{aligned}
& a^{2}-\frac{f_{0}^{(2)}}{f_{0}^{(-1)}} a+\left(\frac{f_{0}^{(1)}}{f_{0}^{(-1)}}-\frac{f_{0}^{(2)}}{f_{0}{ }^{(0)}}\right)=0 \\
& b=\frac{f_{0}^{(1)}}{f_{0}^{(}(-1)}-a \frac{f_{0}^{(0)}}{f_{0}^{(-1)}} \\
& c=a-\frac{f_{0}^{(0)}}{f_{0}^{(-1)}}
\end{aligned}
$$

where the $f_{0}^{(j)}$ are known. Substitution into Equation [38] then supplies the transfer function of the feedback loop to stabilize the system. 
To illustrate application of the stabilization procedure, consider the unstable rocket for which the Satche diagram is shown in Fig. 6. For this example, the parameters have values

$$
\begin{aligned}
& \alpha_{0}=\alpha_{f}=1.0 \\
& P_{0}=P_{f}=1.0 \\
& \begin{array}{ll}
J_{0}=2.0 & J_{f}=1.5 \\
\bar{\gamma}=2.50 & n=0.6
\end{array}
\end{aligned}
$$

The relations of Appendix 2 allow computation of the coefficients in the power expansions of the third-order determinants

$$
\begin{array}{lll}
d_{1}{ }^{(0)}=2.50 & d_{2}{ }^{(0)}=6.25 & d_{0}{ }^{(1)}=3.01 \\
d_{1}^{(1)}=9.75 & d_{2}^{(1)}=6.85 & d_{0}(2)=6.16 \\
d_{1}^{(2)}=9.95 & d_{2}^{(2)}=1.76 & d_{0}^{(3)}=2.89 \\
d_{1}^{(3)}=3.00 & d_{2}^{(3)}=0.02 & d_{0}^{(3)}=0.01
\end{array}
$$

Now if, for small values of $\omega$, the Satche diagram of the system may be moved to that indicated by the broken line in Fig. 16, the rocket motor will be stable against low-frequency oscillations. The curve $g_{2}(i \omega)$ will lie as shown if the $\gamma_{i}$ are chosen

$$
\begin{array}{ll}
\gamma_{0}=-1.25 & \gamma_{2}=-0.25 \\
\gamma_{1}=-2.0 & \gamma_{3}=-1.0
\end{array}
$$

By application of Equations [34] and [37]

$$
\begin{array}{ll}
f_{0}(-1)=-1.41 & f_{0}(1)=-3.87 \\
f_{0}(0)=-2.95 & f_{0}^{(2)}=\mathbf{5 . 0 5}
\end{array}
$$

which fixes the feedback loop for small values of $\omega$. If further the transfer function is chosen to be of the form of Equation [38], the constants $a, b$, and $c$ may be calculated from Equations [40]

$$
\begin{aligned}
& a=-0.8 \\
& b=1.08 \\
& c=1.29
\end{aligned}
$$

$$
\begin{aligned}
& a=-1.29 \\
& b=0.06 \\
& c=0.8
\end{aligned}
$$

the two sets arising through the quadratic equation determining $a$. Choosing the first set (arbitrarily), the transfer function for the feedback loop in the oxidizer line is

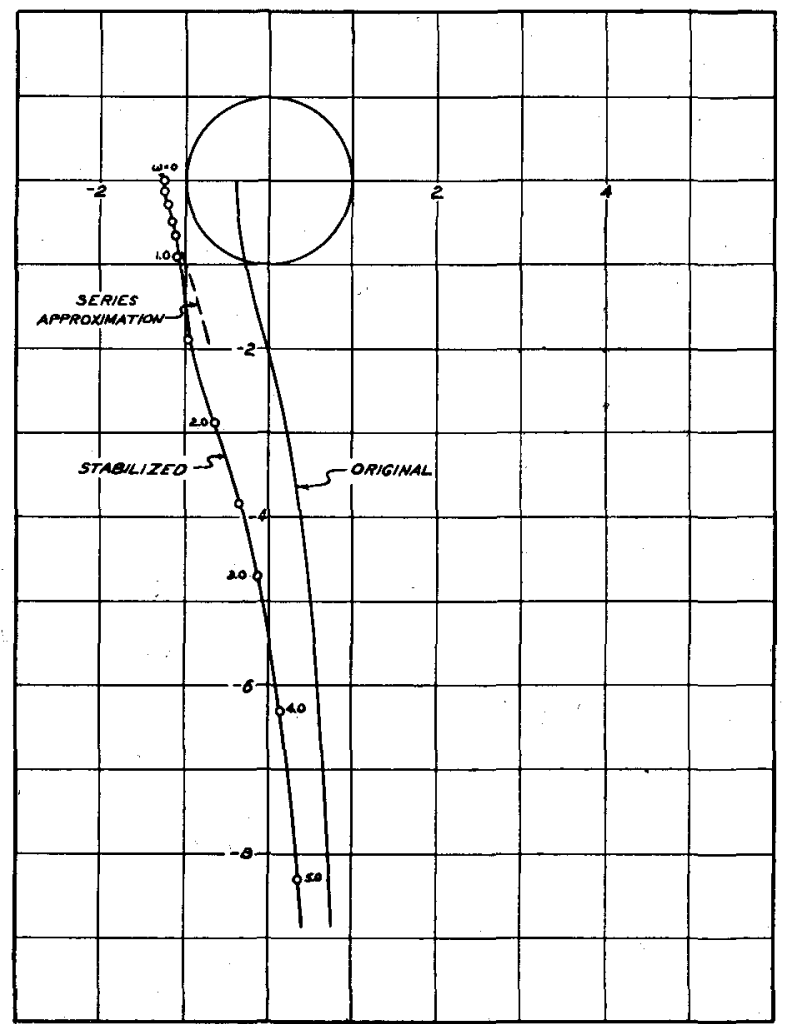

FIG. 16 COMPARISON OF STABILIZED AND UNSTABLE SATCHE DIAGRAMS, $\bar{r}=2.5, n=0.6 ; \alpha_{0}=\alpha_{f}=1.0, P_{0}=P_{f}=1.0, J_{0}=2.0$

$$
J_{f}=1.5
$$

$$
F_{0}(s)=\frac{-1.41}{s} \frac{(1-1.29 s)}{(1+0.8 s)\left(1-1.08 s^{2}\right)}
$$

The Satche diagram for the stabilized motion is now easily computed through use of Equation [30] and the feedback transfer function found above. The result is shown in Fig. 16 along with the original Satche diagram and the power-series approximation for small values of $\omega$. It is of interest to observe from Fig. 16 that the stabilized curve of $g_{2}(i \omega)$ and the corresponding series approximation diverge appreciably only as the value of $\omega$ exceeds unity. Thus the character of the Satche diagram is determined with sufficient accuracy by a few terms of its power-series expansion.

\section{Concluding Remark}

The present analytical results together with the initial work of Tsien (5) demonstrate conclusively that the lowfrequency oscillations of a liquid propellant rocket motor may, in all cases, be stabilized by use of a feedback servo loop. Through use of the Satche diagram and the associated methods which have been described, a projected rocket configuration may be analyzed for possible unstable operation and the requirements for a stabilizing feedback loop determined. The application of these methods lies within common engineering practice. The concept of feedback stabilization removes restrictions on the propellant feed system and injector which would otherwise limit the freedom of the designer, with the consequence that he should be able to focus attention on improving combustion, reducing pumping loss, etc. This freedom is particularly important in the design of a rocket with variable thrust.

\section{APPENDIX 1}

\section{Laplace Transform of the Rocket Stability Equations}

The equation describing the combustion chamber pressure oscillation is

$$
\begin{aligned}
& \frac{d \varphi}{d z}-(1-n) \varphi+n \varphi(z-\delta)+ \\
& {\left[K \mu_{0}(z-\delta-1)-\left(2 K+H+\frac{1}{2}\right) \mu_{0}(z-\delta)\right]-} \\
& \quad\left[K \mu_{f}(z-\delta-1)-\left(2 K+H-\frac{1}{2}\right) \mu_{f}(z-\delta)\right] \ldots
\end{aligned}
$$

while the equations of motion for the liquid in the oxidizer and fuel lines are

$$
\begin{aligned}
J_{0} \frac{d \mu_{0}}{d z}+\left[\frac{1}{\alpha_{0}}\left(P_{0}+\frac{1}{2}\right)+1\right] \mu_{0}+P_{0}+ \\
J_{0} \frac{d^{2} \kappa_{0}}{d z^{2}}+\frac{1}{\alpha_{0}}\left(P_{0}+\frac{1}{2}\right) \frac{d \kappa_{0}}{d z}=0 . \\
J_{f} \frac{d \mu_{f}}{d z}+\left[\frac{1}{\alpha_{f}}\left(P_{f}+\frac{1}{2}\right)+1\right] \mu_{f}+P_{f} \varphi+ \\
J_{f} \frac{d^{2^{2} \kappa_{f}}}{d z^{2}}+\frac{1}{\alpha_{f}}\left(P_{f}+\frac{1}{2}\right) \frac{d \kappa_{f}}{d z}=0 .
\end{aligned}
$$

Denoting the Laplace transforms of $\varphi(z), \mu_{0}(z), \mu_{f}(z), \kappa_{0}(z)$, and $\kappa_{f}(z)$ by $\Phi(s), M_{0}(s), M_{f}(s), K_{0}(s)$, and $K_{f}(s)$, respectively, the transformation of Equations [41], [42], and [43] is straightforward except for the terms involving a time lag. In general, the transform of $f(z-\delta)$ will be

$$
\begin{aligned}
\int_{0}^{\infty} f(z-\delta) e-s z d z & =\int_{-\delta}^{\infty} e-s \delta f(z-\delta) e^{-s(z-\delta)} d(z-\delta) \\
& =e^{-s \delta F(s)-e^{-s \delta} \int_{-\delta}^{0} f(\xi) e-s \xi d \xi}
\end{aligned}
$$

where $F(s)$ is the transform of $f(z)$. As is well known, when the variable is retarded the transform is modified through multiplication by an exponential factor $e^{-s \delta}$ and by the addition of an integral extending over the period of the time lag. 
The equations then transform to

$$
\begin{aligned}
& {\left[s+1-n+n e^{-s \delta}\right] \Phi(s)+} \\
& e^{-s \delta}\left[K e^{-s}-\left(2 K+H+\frac{1}{2}\right)\right] M_{0}(s)- \\
& e^{-s \delta}\left[K e-s-\left(2 K+H-\frac{1}{2}\right)\right] M_{f}(s)= \\
& \varphi(0)+n e^{-s \delta} \int_{-\delta}^{0} \varphi(\xi) e^{-s \xi} d \xi+ \\
& K e-s(1+\delta) \int_{-(1+\delta)}^{0}\left[\mu_{0}(\xi)-\mu_{f}(\xi)\right] e^{-s \xi} d \xi- \\
& (2 K+H) e^{-s \delta} \int_{-\delta}^{0}\left[\mu_{0}(\xi)-\mu_{f}(\xi)\right] e^{-s \xi} d \xi- \\
& \frac{1}{2} e^{-s \delta} \int_{-\delta}^{0}\left[\mu_{0}(\xi)+\mu_{f}(\xi)\right] e^{-s \xi} d \xi \quad[44] \\
& {\left[J_{0} s+1+\frac{1}{\alpha_{0}}\left(P_{0}+\frac{1}{2}\right)\right] M_{0}(s)+P_{0} \Phi(s)+} \\
& {\left[J_{0} s^{2}+\frac{1}{\alpha_{0}}\left(P_{0}+\frac{1}{2}\right) s\right] K_{0}(s)=J_{0} \mu_{0}(0)+J_{0} \frac{d \kappa_{0}}{d z}(0)+} \\
& {\left[J_{0} s+\frac{1}{\alpha_{0}}\left(P_{0}+\frac{1}{2}\right)\right] \kappa_{0}(0) \text {. }} \\
& {\left[J_{f} s+1+\frac{1}{\alpha_{f}}\left(P_{f}+\frac{1}{2}\right)\right] M_{f}(s)+P_{f} \Phi(s)+} \\
& {\left[J_{f} s^{2}+\frac{1}{\alpha_{f}}\left(P_{f}+\frac{1}{2}\right) s\right] K_{f}(s)=J_{f \mu_{f}}(0)+J_{f} \frac{d \kappa_{f}}{d z}(0)+} \\
& {\left[J_{f} s+\frac{1}{\alpha_{f}}\left(P_{f}+\frac{1}{2}\right)\right]}
\end{aligned}
$$

Stability analysis requires only the left-hand sides of these equations. Transient behavior of the rocket system, however, is determined by the complete equations where the righthand sides, in usual problems, consist in the initial conditions of the dependent variables. For systems with time lag, such as the iiquid propellant rocket, Equations [44], [45], and [46] show that in addition to initial conditions evaluated at $z=0$, certain features of the behavior of the system must be known for a period preceding $z=0$ equal in duration to the time lag. Physically this is clear, for due to the combustion time lag, the variation of chamber pressure $\varphi(z)$ cannot be described for $z>0$ unless the behavior of the propellant flow is known for the period $-\delta \leq z \leq 0$.

\section{APPENDIX 2}

\section{Power Series Representation of the Determinants}

$$
D_{1}(s)=d_{1}{ }^{(0)}+d_{1}{ }^{(1)} s+d_{1}{ }^{(2)} s^{2}+d_{1}{ }^{(3)} s^{3}
$$

Since this determinant, Equation [19], is a polynomial, this expression is exact.

$$
\begin{aligned}
& d_{j}^{(0)}=(1-n)\left[1+\frac{1}{\alpha_{0}}\left(P_{0}+\frac{1}{2}\right)\right]\left[1+\frac{1}{\alpha_{f}}\left(P_{f}+\frac{1}{2}\right)\right] \\
& d_{1}^{(1)}=\left[1+\frac{1}{\alpha_{f}}\left(P_{f}+\frac{1}{2}\right)\right]\left[1+\frac{1}{\alpha_{0}}\left(P_{0}+\frac{1}{2}\right)\right]+ \\
& (1-n) J_{0}\left[1+\frac{1}{\alpha_{f}}\left(P_{f}+\frac{1}{2}\right)\right]+ \\
& (1-n) J_{f}\left[1+\frac{1}{\alpha_{0}}\left(P_{0}+\frac{1}{2}\right)\right] \\
& d_{1}^{(2)}=(1-n) J_{0} J_{f}+J_{0}\left[1+\frac{1}{\alpha_{f}}\left(P_{f}+\frac{1}{2}\right)\right]+ \\
& J_{f}\left[1+\frac{1}{\alpha_{0}}\left(P_{0}+\frac{1}{2}\right)\right]
\end{aligned}
$$

$d_{1}{ }^{(3)}=J_{0} J_{f}$

$$
D_{2}(s)=d_{2}{ }^{(0)}+d_{2}^{(1)} s+d_{2}{ }^{(2)} s^{2}+d_{2}{ }^{(3)} s^{3}+\ldots
$$

Referring to Equation [20], the coefficients are $d_{2}{ }^{(0)}=n\left[1+\frac{1}{\alpha_{0}}\left(P_{0}+\frac{1}{2}\right)\right]\left[1+\frac{1}{\alpha_{j}}\left(P_{f}+\frac{1}{2}\right)\right]+$

$$
\begin{gathered}
\frac{1}{2}\left\{P_{0}\left[1+\frac{1}{\alpha_{f}}\left(P_{f}+\frac{1}{2}\right)\right]+P_{f}\left[1+\frac{1}{\alpha_{0}}\left(P_{0}+\frac{1}{2}\right)\right]\right\}- \\
(K+H)\left\{P_{f}\left[1+\frac{1}{\alpha_{0}}\left(P_{0}+\frac{1}{2}\right)\right]-P_{0}\left[1+\frac{1}{\alpha_{f}}\left(P_{f}+\frac{1}{2}\right)\right]\right\} \\
d_{2}^{(1)}=n\left\{J_{0}\left[1+\frac{1}{\alpha_{f}}\left(P_{f}+\frac{1}{2}\right)\right]+\right. \\
\left.J_{f}\left[1+\frac{1}{\alpha_{0}}\left(P_{0}+\frac{1}{2}\right)\right]\right\}+\frac{1}{2}\left\{P_{0} J_{f}+P_{f} J_{0}\right\}+ \\
K\left\{P_{0}\left[1+\frac{1}{\alpha_{f}}\left(P_{f}+\frac{1}{2}\right)\right]-P_{f}\left[1+\frac{1}{\alpha_{0}}\left(P_{0}+\frac{1}{2}\right)\right]\right\}+
\end{gathered}
$$$$
(K+H)\left\{P_{0} J_{f}-P_{f} J_{0}\right\}
$$$$
d_{2}^{(2)}=n J_{0} J_{f}+K\left\{P_{0} J_{f}-P_{f} J_{0}\right\}+
$$$$
\frac{K}{2}\left\{P_{f}\left[1+\frac{1}{\alpha_{0}}\left(P_{0}+\frac{1}{2}\right)\right]-P_{0}\left[1+\frac{1}{\alpha_{f}}\left(P_{f}+\frac{1}{2}\right)\right]\right\}
$$$$
d_{2}^{(3)}=\frac{K}{2}\left\{P_{f} J_{0}-P_{J} J_{f}\right\}+
$$$$
\frac{K}{6}\left\{P_{0}\left[1+\frac{1}{\alpha_{f}}\left(P_{f}+\frac{1}{2}\right)\right]-P_{f}\left[1+\frac{1}{\alpha_{0}}\left(P_{0}+\frac{1}{2}\right)\right]\right\}
$$

$D_{0}(s)=d_{0}{ }^{(0)}+d_{0}^{(1)} s+d_{0}^{(2)} s^{2}+d_{0}^{(3)} s^{3}+d_{0}{ }^{(4)} s^{4}+\ldots$

Referring to Equation [27], the coefficients are

$$
\begin{aligned}
d_{0}^{(0)}= & 0 \\
d_{0}^{(1)}= & \left(K+H+\frac{1}{2}\right) \frac{1}{\alpha_{0}}\left(P_{0}+\frac{1}{2}\right)\left[1+\frac{1}{\alpha_{f}}\left(P_{f}+\frac{1}{2}\right)\right] \\
d_{0}^{(2)}= & \left(K+H+\frac{1}{2}\right)\left\{J_{0}\left[1+\frac{1}{\alpha_{f}}\left(P_{f}+\frac{1}{2}\right)\right]+\right. \\
J_{f} & \left.\frac{1}{\alpha_{0}}\left(P_{0}+\frac{1}{2}\right)\right\}+K \frac{1}{\alpha_{0}}\left(P_{0}+\frac{1}{2}\right)\left[1+\frac{1}{\alpha_{f}}\left(P_{f}+\frac{1}{2}\right)\right] \\
d_{0}^{(3)}= & \left(K+H+\frac{1}{2}\right) J_{0} J_{f}+K\left\{J_{0}\left[1+\frac{1}{\alpha_{f}}\left(P_{f}+\frac{1}{2}\right)\right]+\right. \\
J_{f} & \left.\frac{1}{\alpha_{0}}\left(P_{0}+\frac{1}{2}\right)\right\}-\frac{K}{2} \frac{1}{\alpha_{0}}\left(P_{0}+\frac{1}{2}\right)\left[1+\frac{1}{\alpha_{f}}\left(P_{f}+\frac{1}{2}\right)\right] \\
d_{0}^{(4)}= & K J_{0} J_{f}-\frac{K}{2}\left\{J_{0}\left[1+\frac{1}{\alpha_{f}}\left(P_{f}+\frac{1}{2}\right)\right]+\right. \\
J_{f} & \left.\frac{1}{\alpha_{0}}\left(P_{0}+\frac{1}{2}\right)\right\}+\frac{K}{6} \frac{1}{\alpha_{0}}\left(P_{0}+\frac{1}{2}\right)\left[1+\frac{1}{\alpha_{f}}\left(P_{f}+\frac{1}{2}\right)\right]
\end{aligned}
$$$$
D_{f}(s)=d_{f}^{(0)}+d_{f}^{(1)} s+d_{f}^{(2)} s^{2}+d_{f}^{(3)} s^{3}+d_{f}^{(4)} s^{4}+\ldots
$$

Referring to Equation [28], the coefficients are $d_{f}^{(0)}=0$

$$
\begin{aligned}
d_{f}^{(1)}= & \left(K+H-\frac{1}{2}\right) \frac{1}{\alpha_{f}}\left(P_{f}+\frac{1}{2}\right)\left[1+\frac{1}{\alpha_{0}}\left(P_{0}+\frac{1}{2}\right)\right] \\
d_{f}^{(2)}= & \left(K+H-\frac{1}{2}\right) \\
& \left\{J_{f}\left[1+\frac{1}{\alpha_{0}}\left(P_{0}+\frac{1}{2}\right)\right]+J_{0} \frac{1}{\alpha_{f}}\left(P_{f}+\frac{1}{2}\right)\right\}+ \\
& K \frac{1}{\alpha_{f}}\left(P_{f}+\frac{1}{2}\right)\left[1+\frac{1}{\alpha_{0}}\left(P_{0}+\frac{1}{2}\right)\right] \\
d_{f}^{(3)}= & \left(K+H-\frac{1}{2}\right) J_{0} J_{f}+ \\
K & \left\{J_{f}\left[1+\frac{1}{\alpha_{0}}\left(P_{0}+\frac{1}{2}\right)\right]+J_{0} \frac{1}{\alpha_{f}}\left(P_{f}+\frac{1}{2}\right)\right\}- \\
& \frac{K}{\alpha_{f}}\left(P_{f}+\frac{1}{2}\right)\left[1+\frac{1}{\alpha_{0}}\left(P_{0}+\frac{1}{2}\right)\right] \\
d_{f}^{(4)}= & K J_{0} J_{f}-\frac{K}{2}\left\{J_{f}\left[1+\frac{1}{\alpha_{0}}\left(P_{0}+\frac{1}{2}\right)\right]+\right. \\
J_{0} & \left.\frac{1}{\alpha_{f}}\left(P_{f}+\frac{1}{2}\right)\right\}+\frac{K}{6} \frac{1}{\alpha_{f}}\left(P_{f}+\frac{1}{2}\right)\left[1+\frac{1}{\alpha_{0}}\left(P_{0}+\frac{1}{2}\right)\right]
\end{aligned}
$$

(Continued on page 81) 


\section{Servo-Stabilization of Low-Frequency Oscillations in a Liquid Bipropellant Rocket Motor}

(Continued from page $\%$ )

\section{References}

1 "Stability of Flow in a Rocket Motor," by D. F. Gunder and D. R. Friant, Journal of Applied Mechanics (Trans. ASME), vol. 17,1950 , pp. $327-333$.

2 Discussion of Ref. 1, by M. Yachter, Journal of Applied Mechanics (Trans. ASME), vol. 18, 1951, pp. 114-116.

3 "A Theory of Unstable Combustion in Liquid Propellant
Rocket Motors," by M. Summerfield, Journal of ThE AmERICAN Rocket Societr, vol. 21, September 1951, pp. 108-114.

4 "Aspects of Combustion Stability in Liquid Propellant Rocket Motors, Parts I and II," Journal of THE AmERICAN Rocket Societr, vol. 21, November 1951, pp. 163-178; vol. 22, January-Febuary 1952, pp. 7-16.

5 "Servo-Stabilization of Combustion in Rocket Motors," by H. S. Tsien, Journal of the American Rocket Society, vol. 22, September-October 1952 , pp. 256-262.

6 Discussion by M. Satche of "Stability of Linear Oscillating Systems With Constant Time Lag," by H. I. Ansoff, Journal of Applied Mechanics (Trans. ASME), vol. 16, 1949, pp. 419-420.

7 "Transfer Functions of Rocket Nozzles," by H. S. Tsien, Journal of the American Rocket Society, vol. 22. May-June 1952, pp. 139-143. 